\title{
THE GLOBULAR CLUSTER SYSTEM OF NGC 1399. II. KINEMATICS OF A LARGE SAMPLE OF GLOBULAR CLUSTERS ${ }^{1}$
}

\author{
T. Richtler, ${ }^{2}$ B. Dirsch, ${ }^{2}$ K. Gebhardt, ${ }^{3}$ D. Geisler, ${ }^{2}$ M. Hilker, ${ }^{4}$ M. V. Alonso, ${ }^{5}$ J. C. Forte, ${ }^{6}$ E. K. Grebel, ${ }^{7}$ \\ L. Infante, ${ }^{8}$ S. Larsen, ${ }^{9}$ D. Minniti, ${ }^{8}$ and M. Rejkuba ${ }^{9}$ \\ Received 2002 October 28; accepted 2004 January 8
}

\begin{abstract}
We study the kinematics and dynamics of the globular cluster system of NGC 1399, the brightest elliptical galaxy near the center of the Fornax cluster of galaxies. The observational data consists of medium-resolution spectra, obtained at the Very Large Telescope with FORS2 and the Mask Exchange Unit (MXU). Our sample comprises 468 radial velocities in the magnitude range $20<m_{R}<23$. This is the largest sample of globular cluster velocities around any galaxy obtained so far. Typical velocity uncertainties are $50 \mathrm{~km} \mathrm{~s}^{-1}$, significantly improving on earlier samples. The radial range is $2^{\prime}<r<9^{\prime}$, corresponding to $11 \mathrm{kpc}$ to $50 \mathrm{kpc}$ of galactocentric distance. The shape of the velocity distribution of the sample is compatible with being a Gaussian distribution. However, under moderate error selection, a slight asymmetry is visible between high and low radial velocities. We find bright clusters with radial velocities below $800 \mathrm{~km} \mathrm{~s}^{-1}$, while they are not found at the corresponding highvelocity side above $2000 \mathrm{~km} \mathrm{~s}^{-1}$. There is the possibility that unbound clusters and/or objects in the foreground contaminate the NGC 1399 cluster sample. Under strong error selection, practically no objects are found with velocities lower than $800 \mathrm{~km} \mathrm{~s}^{-1}$ or higher than $2000 \mathrm{~km} \mathrm{~s}^{-1}$. Since the extreme velocities influence the velocity dispersion considerably, uncertainty regarding the exact value of the dispersion remains. With the above velocity limits, we derive a projected velocity dispersion for the total sample of $274 \pm 9 \mathrm{~km} \mathrm{~s}^{-1}$ which within the uncertainties remains constant over the entire radial range. Without any velocity restriction, it increases to $325 \mathrm{~km}$ $\mathrm{s}^{-1}$. Guided by the bimodal color distribution of clusters, we distinguish between red clusters $(C-R>1.6)$ and blue clusters $(C-R<1.6)$, and find velocity dispersions for these groups of $255 \pm 13$ and $291 \pm 14 \mathrm{~km} \mathrm{~s}^{-1}$, respectively, again radially constant. Any possible rotation of either of these cluster populations is below the detection limit, with the exception of a weak signature of rotation for the blue clusters more distant than $6^{\prime}$. Spherical models point to a circular velocity of $419 \pm 30 \mathrm{~km} \mathrm{~s}^{-1}$, assuming isotropy for the red clusters. This value is constant out to $40 \mathrm{kpc}$. The inferred dark halo potential can be well represented by a logarithmic potential. A halo of the NFW type also provides a good fit to the observations. The orbital structure of the clusters can only be weakly constrained. It is consistent with isotropy for the red clusters and a slight tangential bias for the blue clusters. Some mass profiles derived from X-ray analyses do not agree with a constant circular velocity within our radial range, irrespective of its exact value. Interpreting the extreme low radial velocities as space velocities of bound clusters near their pericentric distances would require an extension of the cluster system of at least $200 \mathrm{kpc}$. Implications for formation scenarios of the cluster system are briefly commented on.
\end{abstract}

Key words: dark matter - galaxies: elliptical and lenticular, $\mathrm{cD}$ - galaxies: halos galaxies: individual (NGC 1399) - galaxies: kinematics and dynamics — galaxies: star clusters

\section{INTRODUCTION}

\subsection{General Remarks and Introduction to the Relevant Literature}

To measure the mass profiles of early-type galaxies is not as straightforward as in the case of spiral galaxies, where a more or less orderly rotating disk allows a comparatively easy derivation of the rotation curve (see Sofue \& Rubin 2001 for a review). The inclination of a stellar or $\mathrm{H}$ I disk, which in principle can be measured by the axis ratio, leads to the deprojection of the observed radial velocities, and almost circular orbits directly yield the circular velocities $v_{c}$ :

$$
v_{c}^{2}=\frac{G M(r)}{r},
$$

where $G$ is the constant of gravitation, $r$ is the galactocentric distance, and $M(r)$ is the mass within $r$. Such favorable conditions do not exist for elliptical galaxies. An elliptical galaxy can in reality be axisymmetric, although it appears round in projection. Further complications can result from

\footnotetext{
${ }^{1}$ Based on observations collected at the European Southern Observatory, Cerro Paranal, Chile; ESO program 66.B-0393.

2 Universidad de Concepción, Departamento de Física, Casilla 160-C, Concepción, Chile; tom@coma.cfm.udec.cl.

${ }^{3}$ Department of Astronomy, University of Texas at Austin, 1 University Station, C1400, Austin, TX 78712.

${ }^{4}$ Astronomisches Institut, Universität Bonn, Auf dem Hügel 71, D-53121 Bonn, Germany.

5 Observatorio Astrónomico de Córdoba, Laprida 854, X5000BGR Córdoba, Argentina; CONICET, Argentina; and CNRS UMR 5572, Observatoire Midi-Pyrénées, 14 avenue E. Belin, F-31400 Toulouse, France.

${ }^{6}$ Universidad Nacional de La Plata, Paseo del Bosque s/n, 1900 La Plata, Argentina.

7 Astronomisches Institut, Universität Basel, Venusstrasse 7, CH-4102 Binningen, Switzerland.

${ }^{8}$ Departamento de Astronomíay Astrofísica, Pontificia Universidad Católica, Vicuña Mackenna 4860, Santiago 22, Chile.

${ }^{9}$ European Southern Observatory, Karl-Schwarzschild-Strasse 2, D-85748 Garching, Germany.
} 
nonsymmetric structure due to recent merger events. Moreover, the distribution of stellar orbits is a priori unknown (see de Zeeuw 1994 for an introduction to the dynamics of elliptical galaxies). Therefore, much effort has been devoted to developing more sophisticated stellar dynamical methods in order to derive $M(r)$ for elliptical galaxies from the analysis of the line-of-sight velocity distribution (e.g., Dejonghe 1987; van der Marel \& Franx 1993; Gerhard 1993; Bender, Saglia, \& Gerhard 1994; Rix et al. 1997; Gebhardt et al. 2000).

One of the most important objectives is to prove the existence of dark matter halos and to investigate their structure. For a few X-ray-bright ellipticals, this has been possible by assuming hydrodynamical equilibrium for the hot gaseous halo (e.g., Fabricant \& Gorenstein 1983; Nulsen \& Böhringer 1995 [M87]; Matilsky, Jones, \& Forman 1985 [NGC 4696]; Foreman, Jones, \& Tucker 1985 [13 galaxies]; Irwin \& Sarazin 1996 [NGC 4472]; Mushotzky et al. 1994 [NGC 4636]; Jones et al. 1997 [NGC 1399]; Ikebe et al. 1996 [NGC 1399]; Paolillo et al. 2002 [NGC 1399]). Given the uncertainties and limited applicability of this method (e.g., Buote 2000, 2002; Paolillo et al. 2002), stellar dynamical approaches are very desirable. One faces a further problem in the case of elliptical galaxies: while easily observable objects for determining the rotation curves of spirals sometimes extend to large galactocentric radii (larger than $50 \mathrm{kpc}$ ), the rapidly declining brightness of the light profile of ellipticals rarely allows spectroscopic work beyond 1.5 effective radii. This is a distance at which the effect of a dark halo is only just becoming visible (Kronawitter et al. 2000; Gerhard et al. 2001). However, Kelson et al. (2002), using the Keck telescope, recently achieved measurement of the velocity dispersion of the stellar population of NGC 6166 out to $60 \mathrm{kpc}$.

To extend stellar dynamical investigations to large galactocentric distances, one normally depends on the use of individual dynamical probes in the halos of elliptical galaxies. These probes can be planetary nebulae $(\mathrm{PNe})$ and/or globular clusters (GCs). The observational difficulty is that these objects are faint. There have been some attempts in the past to exploit this kind of dynamical information with $4 \mathrm{~m}$ class telescopes (Arnaboldi et al. 1994 [NGC 1399; PNe]; Grillmair et al. 1994 [NGC 1399; GCs]; Mould et al. 1990 [M87; GCs]), but the sample sizes were small and the accuracy of the radial velocities was low. However, a larger sample of about 100 radial velocities of GCs has been observed by Zepf et al. (2000) for NGC 4472. The advent of large telescopes brought better possibilities in terms of both number and accuracy. A sample of about 550 PNe velocities has been presented by Méndez et al. (2001) for the flattened elliptical galaxy NGC 4607, using the Very Large Telescope (VLT). For NGC 4472, Côté et al. (2003) considerably augmented the sample of GC velocities to $260 \mathrm{GCs}$, using the Keck telescope.

The demands for a dynamically meaningful sample are severe: numerical simulations have shown that several hundred, perhaps even thousand, radial velocities are required if one intends to fit the potential and the phase space distribution simultaneously (e.g., Merritt \& Tremblay 1993). The potential and the orbital properties of dynamical probes are degenerate with respect to radial velocities, so one has to make specific assumptions concerning symmetry and isotropy/anisotropy if one wants to use the observed velocity dispersions to infer the mass profile with a smaller sample.

M87, the most prominent GC rich elliptical galaxy in the northern hemisphere, has in recent years become the target for ambitious studies regarding its globular cluster system (GCS).
Cohen \& Ryzhov (1997) and Cohen (2000) observed about 200 radial velocities, using the Keck telescope. This sample has been improved and enlarged to about 300 velocities by Côté et al. (2001) and Hanes et al. (2001), but even this large data set has rather been used to explore the kinematics of the globular cluster system in relation to its population properties than to derive $M(r)$.

In the southern hemisphere, the technological preconditions for such a demanding project have been considerably improved by the advent of sophisticated spectroscopic equipment at large telescopes, which allows the observations of hundreds of velocities with satisfactory accuracy within a reasonable observing time. The prime natural target is NGC 1399 in the Fornax Cluster, given its proximity and populous GCS.

\subsection{NGC 1399}

NGC 1399 is a particularly attractive galaxy with regard to the study of dark matter halos. It is the brightest elliptical galaxy in the nearest galaxy cluster in the southern hemisphere, the Fornax Cluster (e.g., Drinkwater, Gregg, \& Colless 2001, and references therein). It has a distinguished location near the center of this cluster. In the literature, it has sometimes been labeled as the central $\mathrm{cD}$ galaxy. It is not within the scope of this paper to expand on these characteristics, but see Dirsch et al. (2003, hereafter Paper I), who present the first wide-field CCD study, for a discussion as to why this label is questionable.

Since the distance is one of the most fundamental parameters in all analyses, we include a brief summary of what is known of the distance of NGC 1399 and the Fornax Cluster. A compilation of older work on the Fornax Cluster using several methods can be found in Richtler et al. (1999), resulting in a mean distance modulus of $31.36 \pm 0.06$. Ferrarese et al. (2000) calibrated several methods and compiled distances for 10 early-type Fornax galaxies, giving 31.44 (with a very small formal error) as the average. They list NGC 1399 with $31.31 \pm 0.09$. The catalog of surface brightness fluctuation measurements by Tonry, Dressler, \& Blakeslee (2000) includes 16 galaxies in the core of the Fornax Cluster for which one derives a mean modulus of $31.50 \pm 0.05$, and NGC 1399 is given as $31.50 \pm 0.16$. Note that none of the distance uncertainties include the uncertainty in the absolute scale, which is around $\pm 0.16 \mathrm{mag}$, mainly due to the LMC distance uncertainty. This gives some freedom in the choice of the distance modulus. A value of 31.40 lies within the uncertainty interval of all measurements made so far, and we will adopt it here. The corresponding distance is then $19 \mathrm{Mpc}$.

NGC 1399 offers a wealth of GCs. The total number from recent wide-field photometry is about 7000 (Paper I). The central galaxies in galaxy clusters often have the highest specific frequencies of GCSs occurring among elliptical galaxies. The specific frequency is defined as $S_{N}=N 10^{0.4\left(M_{V}+15\right)}$, where $N$ is the total number of GCs and $M_{V}$ the absolute $V$ magnitude of the host galaxy (see Elmegreen 2000 for a review). The classical value for NGC 1399 is 10 or even larger (e.g., Wagner, Richtler, \& Hopp 1991; Bridges, Hanes, \& Harris 1991). However, several recent studies (Paper I; Ostrov, Forte, \& Geisler 1998) derive a value of about 6 . This change is mainly due to a better account of the total luminosity of NGC 1399, as has been also mentioned by McLaughlin (1999a). Although still high for a giant elliptical, there are also noncentral galaxies such as NGC 4728 (Harris \& van den Bergh 1981), NGC 4636 (Kissler-Patig et al. 1994), or IC 4051 (Woodworth \& Harris 2000), which reportedly reach or even surpass this value. 
Dynamical studies of the stellar body of NGC 1399 have been performed by Bicknell et al. (1989), Graham et al. (1998), Saglia et al. (2000), and Kronawitter et al. (2000). Regarding the cluster system of NGC 1399, the situation was still unsatisfactory. Grillmair et al. (1994) obtained 46 velocities for GCs in NGC 1399; Kissler-Patig et al. (1999) augmented this sample to 76. Spectroscopy for smaller samples of GCs, with the goal of measuring ages and abundances rather than studying the dynamics, has been published by Mieske, Hilker, \& Infante (2002) and Forbes, Beasley, \& Brodie (2001).

This paper is the second in a series on the cluster system of NGC 1399. Details of the data reduction and the data set are presented in a parallel paper (Dirsch et al. 2004, hereafter Paper III). New wide-field photometry in Washington $C$ and Kron-Cousins $R$, from which we selected the cluster candidates and derived the properties of the cluster system needed in dynamical studies, is found in Paper I. These properties are the deprojected luminosity profile of NGC 1399 in $R$, which has been determined to

$$
\left(L_{\odot} / \mathrm{pc}^{3}\right)=101(1+r / 221 \mathrm{pc})^{-2.85}
$$

and the power-law exponents for the deprojected number density profiles of blue $(1.2<C-R<1.6)$ and red clusters $(1.6<C-R<2.2)$, which are $-1.8 \pm 0.1$ and $-2.64 \pm 0.1$, respectively.

The scope of the present paper is to show those results emerging from the data set that can be derived without sophisticated dynamical modeling, which is left to a forthcoming paper (Gebhardt et al. 2004). We briefly present the observations $(\S 2)$, present the morphology of the velocity distribution, investigate the rotation properties, the observed (projected) velocity dispersions and their radial dependence, and their significance for different cluster populations (all in $\S 3$ ), interpret them in the context of a simple spherical model (§ 4$)$, and discuss the results in comparison with the literature. Finally, we comment on formation scenarios of the cluster system $(\S 5)$. Section 6 contains our conclusions and outlook for further work.

\section{OBSERVATIONS AND VELOCITY MEASUREMENTS}

Paper III provides the details of the slit mask preparation, the observations and reduction, and the database. Here we only give a summary of the observations and the radial velocity measurements.

\subsection{Observations}

Paper I provides the candidate selection based on wide-field photometry in Washington $C$ and Kron-Cousins $R$ obtained with the CTIO $4 \mathrm{~m}$ MOSAIC system. The spectroscopic observations have been performed in the period 2000 November 30 to December 2 at the VLT of the European Southern Observatory at Cerro Paranal with Unit Telescope 2 (Kueyen). The instrument was the focal reducer FORS2 equipped with the Mask Exchange Unit (MXU), which allows the use of up to ten slit masks during the night. ${ }^{10}$ The grism in use, for all but one mask, was $600 \mathrm{~B}$, giving a spectral resolution of about $2.5 \AA$, based on the widths of the arc calibration lines. For one mask, we used grism $300 \mathrm{~V}$, which gave a resolution of about $5 \AA$. The wavelength calibration was

\footnotetext{
${ }^{10}$ For details see http://www.eso.org/instruments/fors/userman/.
}

based on He-Ar lamp spectra. The spectral range covered about $2000 \AA$. However, depending on the position of a given slit in the mask, the limiting wavelengths can be as short as $3500 \AA$ and as long as $6500 \AA$. In most cases, the grism efficiency degraded the signal-to-noise ratio $(\mathrm{S} / \mathrm{N})$ shortward of $3800 \AA$ drastically, so this region could not be used.

We exposed 13 masks (exposure times were either $1 \times$ or $2 \times 45$ minutes per mask). The FWHM of objects along the slit ranged from 0.6 to 0.9 . The total number of spectra obtained is 1462. This sample is composed of 531 sky spectra, 512 spectra of cluster candidates, 190 spectra of point sources of unknown nature at the time of mask preparation (stars, unresolved galaxies or clusters not matching the selection criteria), 176 galaxies, and 53 "bright" objects, mainly stars needed to adjust the mask. Since some objects (about 80 ) have been observed in two different masks in order to assess the errors, the total number of objects is smaller by this number than the number of spectra. We anticipate here that the final sample of cluster velocities comprises 468 objects.

\subsection{Velocities}

We used two different techniques to measure the radial velocities: a cross-correlation technique and direct measurements of line positions.

For cross-correlation, we needed a template with a high $\mathrm{S} / \mathrm{N}$ and a spectrum resembling that of a globular cluster. NGC 1399 itself is not suitable because of its high velocity dispersion. We therefore chose NGC 1396 as a template, which has a high $\mathrm{S} / \mathrm{N}$ (about 30) and, as a dwarf elliptical galaxy, has a spectrum similar to that of a metal-rich globular cluster. Regarding line measurements, we defined a set of about 20 easily identifiable features (for which only a few remained in faint spectra) and used the IRAF task rvidlines for measuring the radial velocities. In some cases, it was only possible to get a velocity by cross-correlation and not by lines. For a more detailed discussion of the errors, we refer the reader to Paper III. Figure 1 plots our uncertainties against the cluster magnitude. For comparison we also include the M87 sample from Hanes et al. (2001).

\section{RESULTS}

\subsection{Total Numbers, Projected Distribution, and Absolute Velocities}

The total number of clusters for which we have velocities is 508. However, there are 40 objects for which we could not find a correlation velocity, and 49 objects for which we could not derive a velocity by line positions because of low $\mathrm{S} / \mathrm{N}$. Here we prefer to use only the correlation velocities, for reasons of homogeneity and because they have smaller uncertainties. However, in the case of low $\mathrm{S} / \mathrm{N}$, the uncertainty may be underestimated, since we used the width of the correlation peak to estimate the error.

For our template galaxy NGC 1396, we derive a heliocentric radial velocity of $815 \pm 8 \mathrm{~km} \mathrm{~s}^{-1}$. This is marginally in agreement with the value of $857 \pm 37 \mathrm{~km} \mathrm{~s}^{-1}$ given by Da Costa et al. (1998), not in agreement with $894 \pm 29 \mathrm{~km} \mathrm{~s}^{-1}$ from the RC3 (de Vaucouleurs et al. 1991), and not in agreement with $882 \pm 39 \mathrm{~km} \mathrm{~s}^{-1}$ from Hilker, Infante, \& Richtler (1999). Better agreement is reached with $836 \pm 32 \mathrm{~km} \mathrm{~s}^{-1}$, the value from the Nearby Early-type Galaxies Survey (ENEAR; Wegner et al. 2003). Our value is in very good agreement with $808 \pm 22 \mathrm{~km} \mathrm{~s}^{-1}$ given by Drinkwater et al. (2001a). As for NGC 1399 itself, one finds nine measurements of its radial 


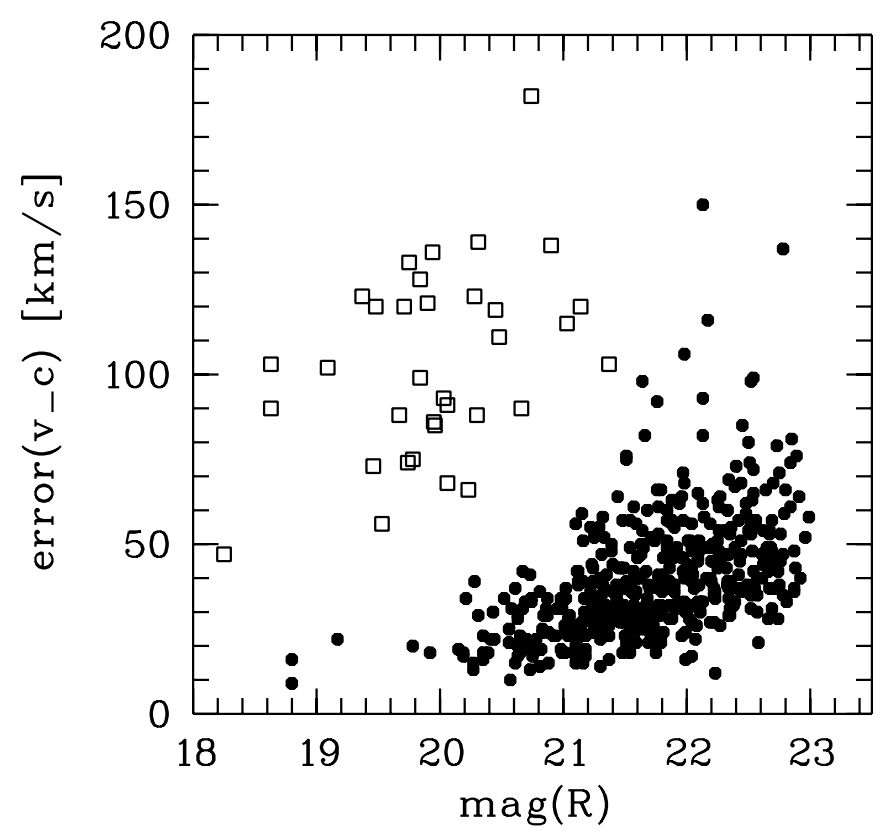

FIG. 1.-Plot of the relation between the $R$ magnitude of the clusters and the uncertainties of their radial velocities (filled circles). For comparison, also plotted (squares) are the magnitudes and velocity uncertainties for the sample of M87 clusters of Hanes et al. (2001), for which errors below $200 \mathrm{~km} \mathrm{~s}^{-1}$ are quoted.

velocity with quoted uncertainties consulting the NASA/IPAC Extragalactic Database. After skipping two of them (one has a discrepant value and the other a large uncertainty of $200 \mathrm{~km} \mathrm{~s}^{-1}$ ), the weighted mean value is $1442 \pm 9 \mathrm{~km} \mathrm{~s}^{-1}$. The ENEAR value is $1425 \pm 15 \mathrm{~km} \mathrm{~s}^{-1}$. Our mean radial velocity of the entire cluster sample is $1441 \pm 15 \mathrm{~km} \mathrm{~s}^{-1}$, so we are confident of our absolute velocity calibration.

Figure 2 shows the distribution on the sky of all clusters for which velocities could be measured by cross-correlation. Since not all prepared masks could be observed, some gaps remain, most strikingly in the southeast quadrant. Because

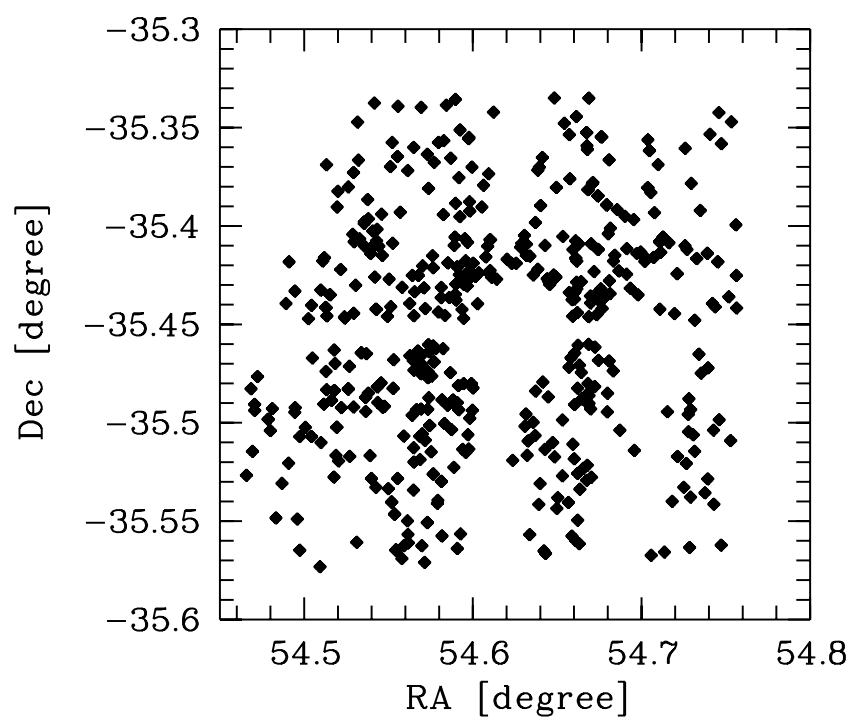

FIG. 2.-Plot of the distribution of clusters on the sky for which correlation velocities are available. Note that the $x$-axis is right ascension, and one must multiply it with the cosine of the declination to get the same projected scale as the $y$-axis. of the increasing background of the galaxy light, we avoided targets with galactocentric distances smaller than about $2^{\prime}$. Our most distant object lies almost $10^{\prime}$ from the center of NGC 1399.

\subsection{Red and Blue Clusters}

Because we use the bimodality in the color distribution of clusters to divide them into two populations, we briefly comment on the color-magnitude diagram (CMD). A bimodal color distribution is a frequent feature of globular cluster systems (for recent work, see Larsen et al. 2001 and Kundu et al. 2001). In the case of NGC 1399, it has already been observed by Kissler-Patig et al. (1997), Forbes et al. (1998), and Ostrov et al. (1998). Figure 3 gives the Washington CMD for almost all of our spectroscopic sample. For 32 clusters, we have no photometry for various reasons, mainly because these objects were located in gaps of the undithered MOSAIC frames.

In this CMD, the color bimodality is not visible. It is, however, very striking in the complete photometry of Paper I. But there also, the bimodality disappears for objects brighter than $R=21 \mathrm{mag}$, and the majority of these bright clusters are found at intermediate colors.

We note (as discussed in more detail in Paper I) that the bimodality does not necessarily imply the existence of two and only two different populations: only the blue (metal-poor) peak lies on the linear part of the color-metallicity relation and corresponds to a peak in metallicity. On the red (metal-rich) end, the relation between color and metallicity becomes nonlinear and finally flat. This causes a peak at an almost universal color under a broad range of metallicity distributions and is not easily interpretable as a corresponding peak in metallicity.

Whatever the nature of the bimodality is, it is well known from many studies that metal-rich clusters behave differently from metal-poor clusters. For example, the blue clusters tend to

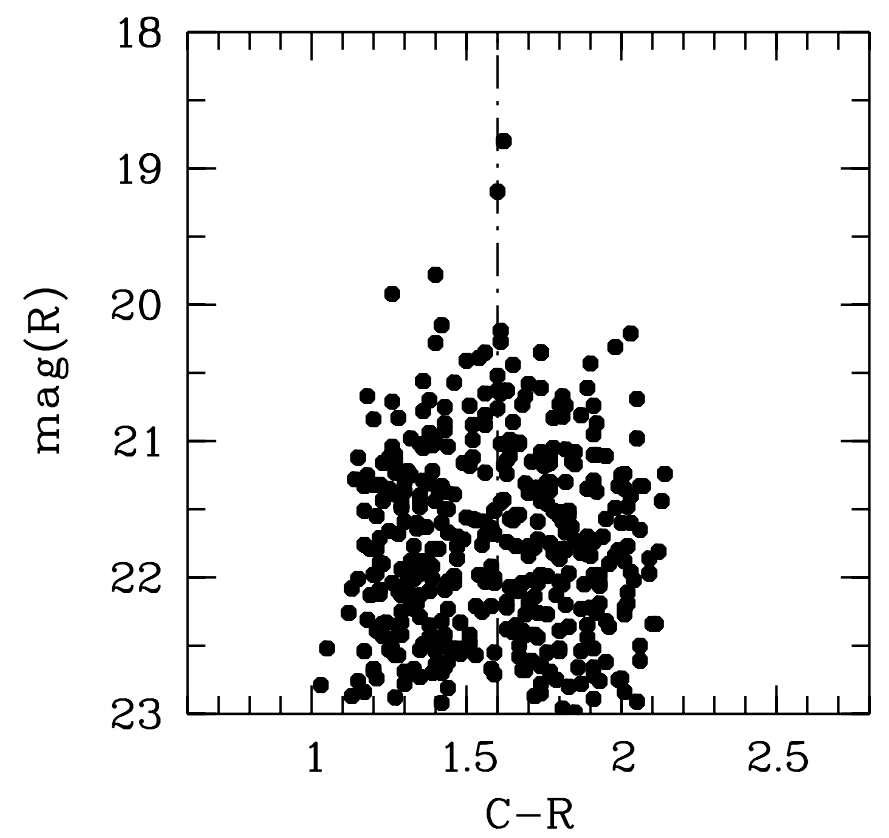

Fig. 3.-Plot of the color-magnitude diagram for the sample of Fig. 2, showing $C-R$ (Washington $C$, Kron $R$ ) vs. $R$. The bimodal color distribution of the NGC 1399 cluster system is more pronounced in a larger and fainter sample (see Paper I) except for the bright clusters, which do not show a bimodal distribution. We define the limit distinguishing red and blue clusters to be $C-R=1.6$. 
show a flatter spatial distribution than the red clusters (see Harris 2001 for a review). Furthermore, the kinematics tend to be different between the red and blue clusters. In the M87 cluster system, for instance, metal-rich clusters seem to show preferentially radial orbits, while the orbits of metal-poor clusters are more tangentially biased (Côté et al. 2001, but see our corresponding remarks in $\S 5$ ). Following Paper I, we define $C-R=1.6$ as the distinguishing color between red and blue clusters. According to the Washington calibration of Harris \& Harris (2002), this color corresponds to a metallicity of $[\mathrm{Fe} / \mathrm{H}]=-0.6 \mathrm{dex}$.

\subsection{Velocity Distribution of the Entire Sample}

In Figure 4, we show the velocity distribution of the entire sample of 468 objects (top left panel). The bin size has been chosen to be $70 \mathrm{~km} \mathrm{~s}^{-1}$, larger than the mean error, but still of satisfactory resolution. The top right panel shows an errorselected sample $\left(<50 \mathrm{~km} \mathrm{~s}^{-1}\right)$, demonstrating that the distribution keeps its shape under error selection. The bottom panels show an inner sample (left) and an outer sample (right). Obvious foreground stars with radial velocities of less than $300 \mathrm{~km} \mathrm{~s}^{-1}$ are omitted, but are listed in the accompanying data paper. For the full sample, the mean velocity and its standard deviation are 1441 and $329 \mathrm{~km} \mathrm{~s}^{-1}$, respectively (note that the velocity dispersions will turn out slightly different). This should be compared with the values (1429 \pm 45 and $373 \mathrm{~km} \mathrm{~s}^{-1}$ ) given by Kissler-Patig et al. (1999) from their sample of 76 GCs.

At first glance, the shape of the distribution does not look very Gaussian. A Kolmogorov-Smirnov (K-S) test gives a probability of only 0.1 that it is drawn from a Gaussian with the above center and dispersion. The maximum probability of 0.57 is given by a dispersion of $298 \mathrm{~km} \mathrm{~s}^{-1}$. One nonGaussian feature is that the distribution does not peak at its mean velocity, a fact also seen in the sample of Kissler-Patig et al. (1999). In their Figure 2, a second peak appears at a velocity of $1800 \mathrm{~km} \mathrm{~s}^{-1}$. A small peak is found at this velocity in our sample as well. It remains with lower bin width, but its significance is doubtful. The only striking feature is that it is made up of predominantly blue clusters, which are nevertheless evenly distributed over the whole field. Figure 4 might suggest the existence of two peaks around the systemic velocity, caused by rotation of a subsample. As we will see, no further evidence for this is found. Furthermore, the distribution looks asymmetric, in that it seems to contain a larger population of clusters on the low-velocity side. This as well

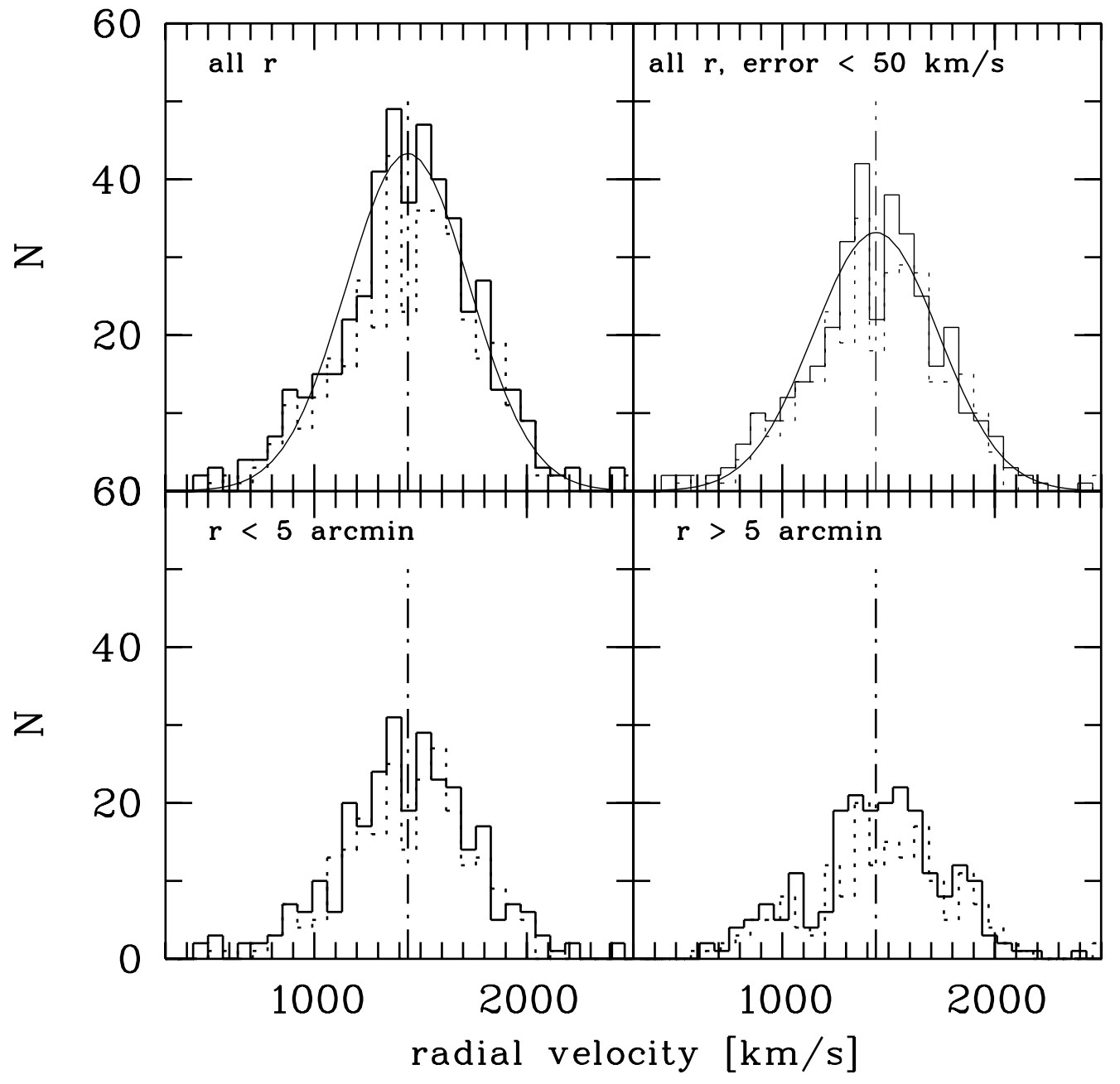

FIG. 4.-Velocity histograms of the entire sample (top left), an error selected sample (top right), an inner sample (bottom left), and an outer sample (bottom right).

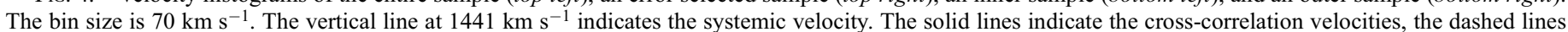

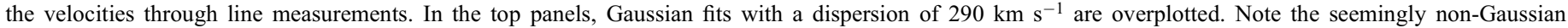

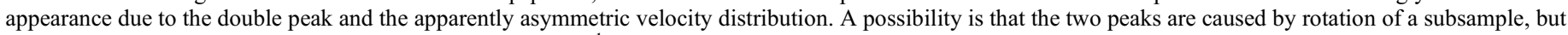
no support for this has been found. The peak at $1800 \mathrm{~km} \mathrm{~s}^{-1}$ is also apparent in the sample of Kissler-Patig et al. (1999). 
is marginally apparent in Kissler-Patig et al. (their Fig. 2), although the authors do not mention it. To compare the lowvelocity part (lower than the systemic velocity) with the high-velocity part, we mirrored both parts to produce two symmetric distributions and performed K-S tests. While the high velocities are drawn from a Gaussian with $1440 \mathrm{~km} \mathrm{~s}^{-1}$ as systemic velocity and $308 \mathrm{~km} \mathrm{~s}^{-1}$ as dispersion with a probability of 0.99 , the K-S test finds a probability of 0.63 for the low velocities using the same parameters.

However, simple simulations of Gaussian distributions, having the same number of objects, quickly show that a K-S test frequently gives low probabilities. For the purpose of illustration, Figure 5 shows six simulations with 470 objects each. The parent populations are Gaussians with $1440 \mathrm{~km} \mathrm{~s}^{-1}$ as the "systemic" velocity and a dispersion of $300 \mathrm{~km} \mathrm{~s}^{-1}$. Indicated are the probabilities returned by a K-S test (we used the command KSTEST/1SAMPLE under MIDAS). "Peaks" occur frequently, and one would conclude that the significance of peaks in Figure 4 is doubtful as well.

Figure 6 plots the correlation velocities against projected radii in arcminutes. The top panel shows all clusters, while the middle panel selects those for which both correlation and line velocities are available and differ by less than $100 \mathrm{~km}$ $\mathrm{s}^{-1}$. Again, it appears as if the low-velocity wing would be more extended, but also that the limit at high velocities at $2000 \mathrm{~km} \mathrm{~s}^{-1}$ is sharper than at low velocities. The bottom panel shows those objects for which the difference between correlation and line velocities is less than $50 \mathrm{~km} \mathrm{~s}^{-1}$. These are our best velocities, and here a low-velocity limit at about the symmetric velocity becomes visible as well. Although one wants to determine the velocity dispersion with as many objects as possible, the existence of velocity limits symmetric to the systemic velocity suggests that the velocity dispersion should be determined within these limits. After we have discussed the dynamics, we will come back to this point and see that this sharp borders are about $140 \mathrm{~km} \mathrm{~s}^{-1}$ above the circular velocity $\left(415 \mathrm{~km} \mathrm{~s}^{-1}\right)$. Distinctly higher velocities can only be produced by objects on very elongated orbits near their pericenters and for which the radial velocity measures approximately the space velocity. These are expected to be rare, leaving more quantitative statements to a theoretical model of the system. Possibilities are unbound objects and/or objects in the foreground, which will be discussed later on.

Looking for further properties that may distinguish low and high velocities, we find their luminosity distribution to be quite different. With respect to velocities, the cluster candidates have been selected randomly, and the distribution of magnitudes is accordingly expected to be similar and symmetric around the systemic velocity. Figure 7, in which the $R$ magnitudes are plotted versus the radial velocities, demonstrates that this is not the case. Vertical dashed lines indicate the systemic velocity (middle line), a velocity of $2000 \mathrm{~km} \mathrm{~s}^{-1}$, and the corresponding symmetric low velocity of $880 \mathrm{~km} \mathrm{~s}^{-1}$. While the number of objects fainter than $R=22$ mag statistically agree on the high- and low-velocity sides, it is striking that only one
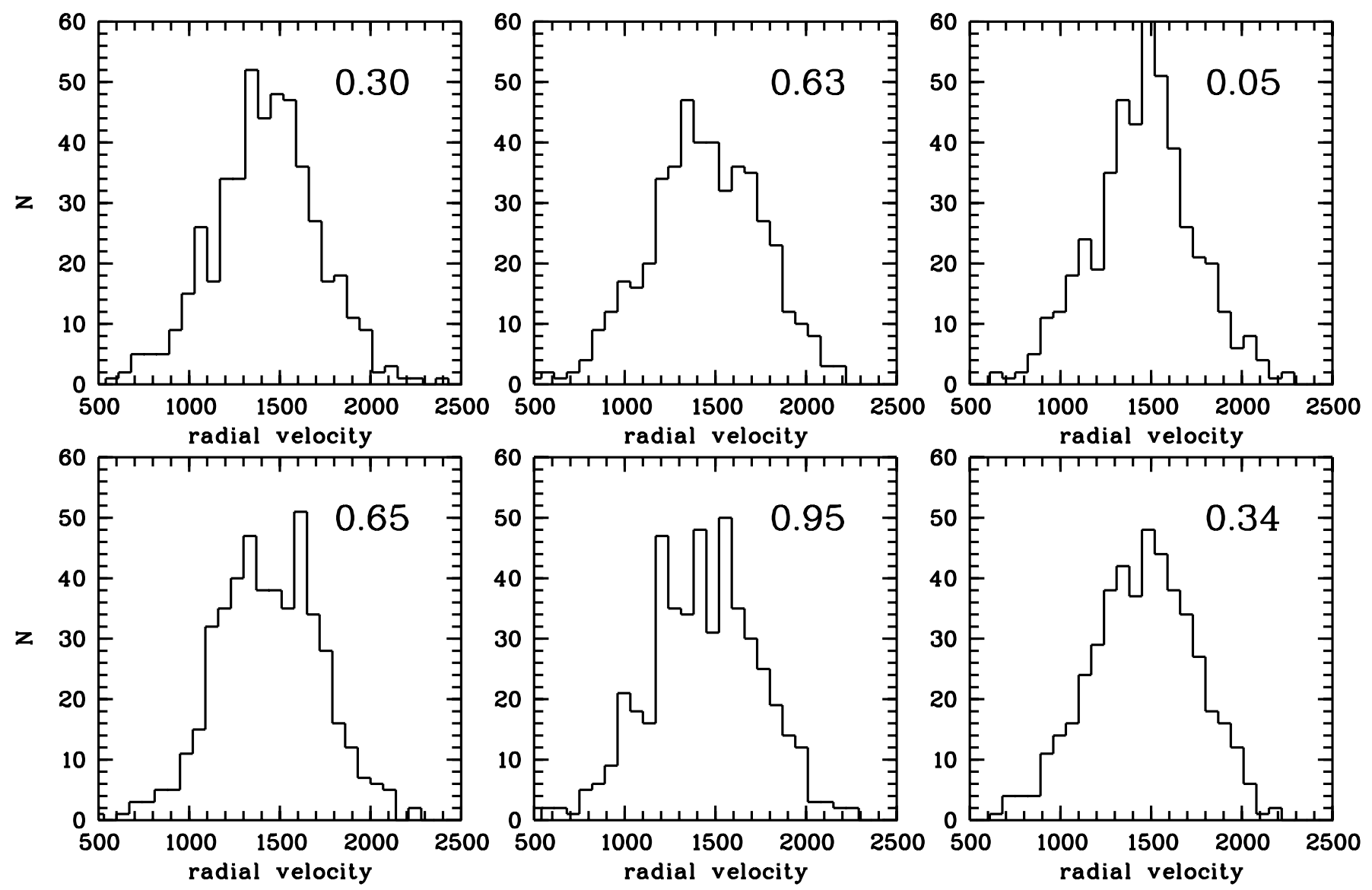

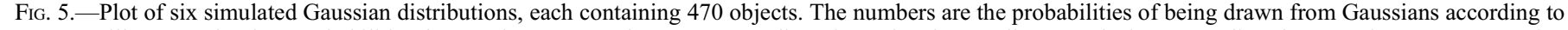

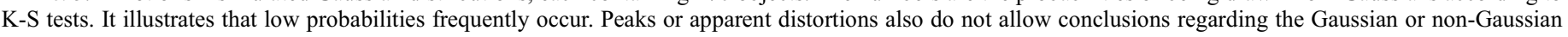
nature. 


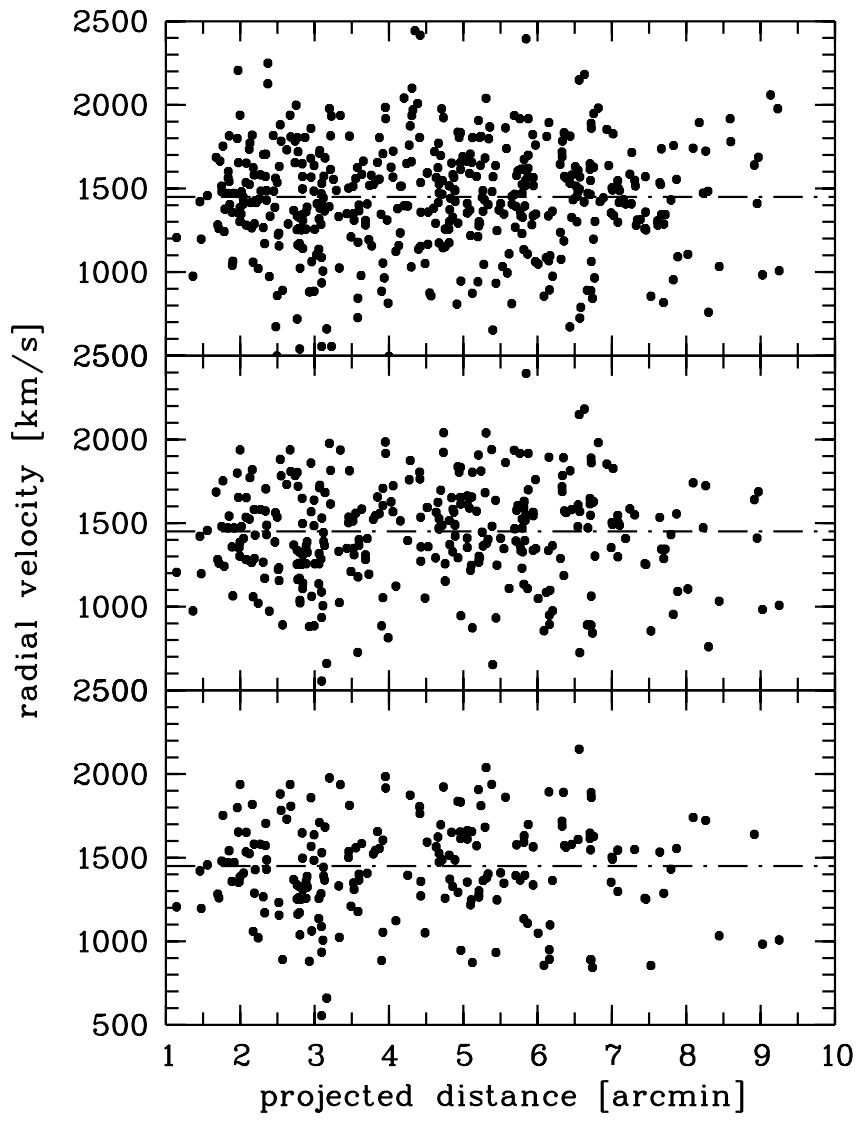

FIG. 6.-Top panel shows the velocities vs. projected galactocentric distance for all clusters. The dashed-dotted line is the systemic radial velocity of NGC 1399. In the middle panel, we selected those clusters for which the difference between correlation velocity and velocities measured by lines amounts to less than $100 \mathrm{~km} \mathrm{~s}^{-1}$. The lower panel shows those objects for which this difference is less than $50 \mathrm{~km} \mathrm{~s}^{-1}$. The upper limit at about $2000 \mathrm{~km}$ $\mathrm{s}^{-1}$ is already discernable in the unselected sample. The symmetric lowvelocity limit at about $800 \mathrm{~km} \mathrm{~s}^{-1}$ shows up only after a strict error selection.

object at $R=21.6 \mathrm{mag}$ is found with a velocity (marginally) higher than $2000 \mathrm{~km} \mathrm{~s}^{-1}$, while there are 10 objects with velocities of $880 \mathrm{~km} \mathrm{~s}^{-1}$ or lower with magnitudes brighter than $R=22 \mathrm{mag}$. This is in spite of the fact that for both low and high velocities, we are biased toward bright objects.

The suspicion may arise that some low-velocity objects could belong to a population in the foreground, because such asymmetry should not be present in a spherical and more or less isotropic system. In that case, one would expect these objects not to be concentrated around NGC 1399. Unfortunately, our sample is not very suitable to searching for differences in the radial distribution of subsamples, since the coordinate distribution is as much determined by the mask and slit positioning as by the true spatial distribution. Selecting the objects with, say, less than $900 \mathrm{~km} \mathrm{~s}^{-1}$ results in 34 clusters, which must be regarded as the minimum for senseful statistical statements. We examined the radial cumulative distribution of these clusters together with a comparison sample of velocities higher than $1300 \mathrm{~km} \mathrm{~s}^{-1}$ and found no difference.

\subsection{Velocity Histograms of Red and Blue Clusters}

Figure 8 shows the velocity histograms of the red and the blue clusters. The total number now is 437 , because we do not have colors for all objects. The distributions of red and blue

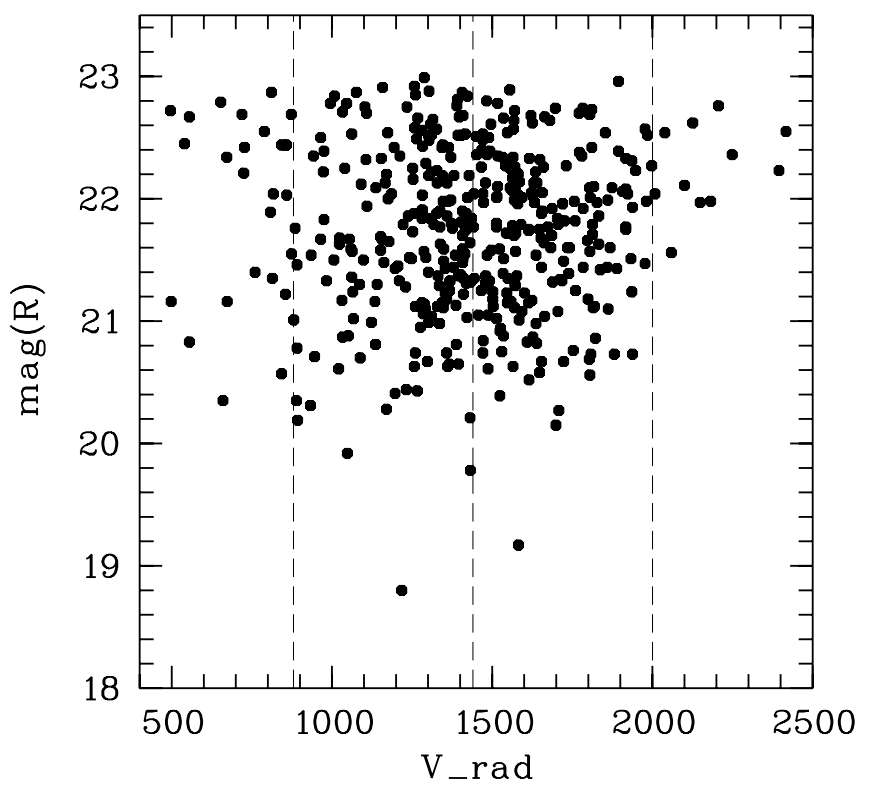

FIG. 7. $-R$ magnitudes vs. radial velocities. This sample is somewhat smaller because there is no photometry available for about 30 clusters. The vertical lines are at $2000 \mathrm{~km} \mathrm{~s}^{-1}$ (to roughly indicate a velocity above which only faint objects are found), at $1440 \mathrm{~km} \mathrm{~s}^{-1}$ (the mean velocity of the entire sample), and at $880 \mathrm{~km} \mathrm{~s}^{-1}$, the corresponding velocity on the low-velocity side. It is striking that there are no bright objects at high velocities, while there are some at very low velocities (the faint objects may have individually uncertain velocities). This may indicate that some of the bright clusters actually are located in the foregound.

clusters look rather different. The formal standard deviations of the velocities are 362 and $289 \mathrm{~km} \mathrm{~s}^{-1}$ for the blue and red clusters, respectively. As will be discussed later on, this difference is attributed to their different surface density profiles. However, these values are larger than those we derive as velocity dispersions, pointing to the difficulty in deciding which is the correct sample for the determination of the velocity dispersion. With this sample, which also includes the extreme velocities, we probably overestimate the true velocity dispersion by the existence of objects that may not belong to the NGC 1399 system.

The detailed morphology of these histograms is somewhat dependent on the binning. However, the peak of the blue clusters near $1800 \mathrm{~km} \mathrm{~s}^{-1}$ is stable against varying the bin width, and it is now clear that it is indeed composed of preferentially blue clusters. In addition, the peak at $1550 \mathrm{~km}$ $\mathrm{s}^{-1}$ remains for smaller bin widths than the present $70 \mathrm{~km} \mathrm{~s}^{-1}$. Whether the central double peak of the red clusters is caused by rotation is investigated later on.

The different velocity dispersions are also visible in Figure 9, which shows radial distance vs. velocity for the red and blue samples. Furthermore, it appears that for distances smaller than about $3^{\prime}$, the velocity dispersion decreases toward the center, perhaps somewhat more distinctly for the red clusters than for the blue clusters. We will come back to this point when discussing the velocity dispersions.

\subsection{Rotation and Azimuthal Behavior}

A fundamental kinematic question for our sample is whether we find signatures of rotation and whether these are different for different subpopulations of globular clusters. The diagnostic diagram that we have to analyze is a plot of radial velocities versus the position angle. Côté et al. (2001) give a 


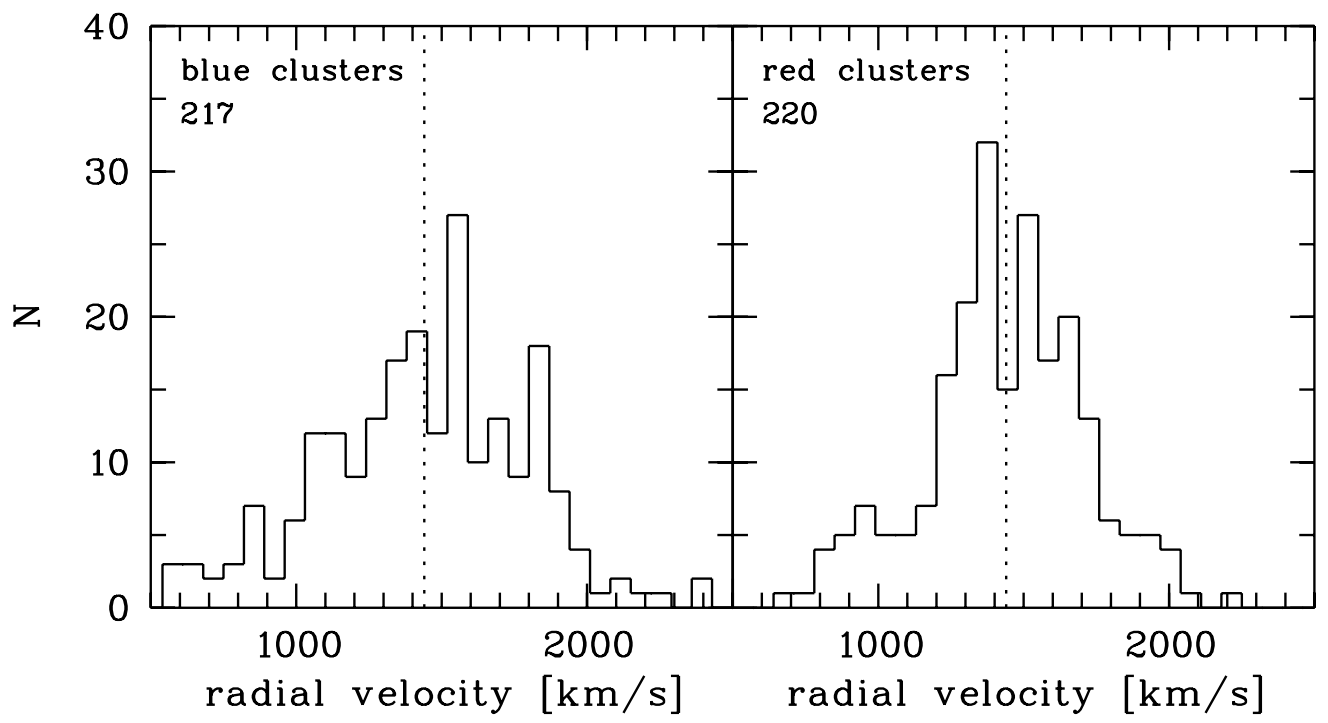

Fig. 8.-Velocity distribution (correlation velocities) for the red and the blue clusters separately. The lower velocity dispersion of the red clusters is discernible. Both distributions show the extended wing toward low velocities. The systemic velocity is indicated by the dotted vertical line.

useful discussion of the relation between the intrinsic and projected rotational velocity field of a spherical system, and we do not repeat that here. If the intrinsic rotation velocity field is stratified on spheres, and the galaxy is not seen poleon, we measure radial velocities that depend sinusoidally on the azimuth angle. Therefore, we fit the relation

$$
v_{r}(\Theta)=v_{\text {sys }}+A \sin \left(\Theta-\Theta_{0}\right),
$$

where $v_{r}$ is the projected radial velocity at the azimuth angle $\Theta$, $v_{\text {sys }}$ is the systemic velocity, and $A$ is the rotation amplitude.

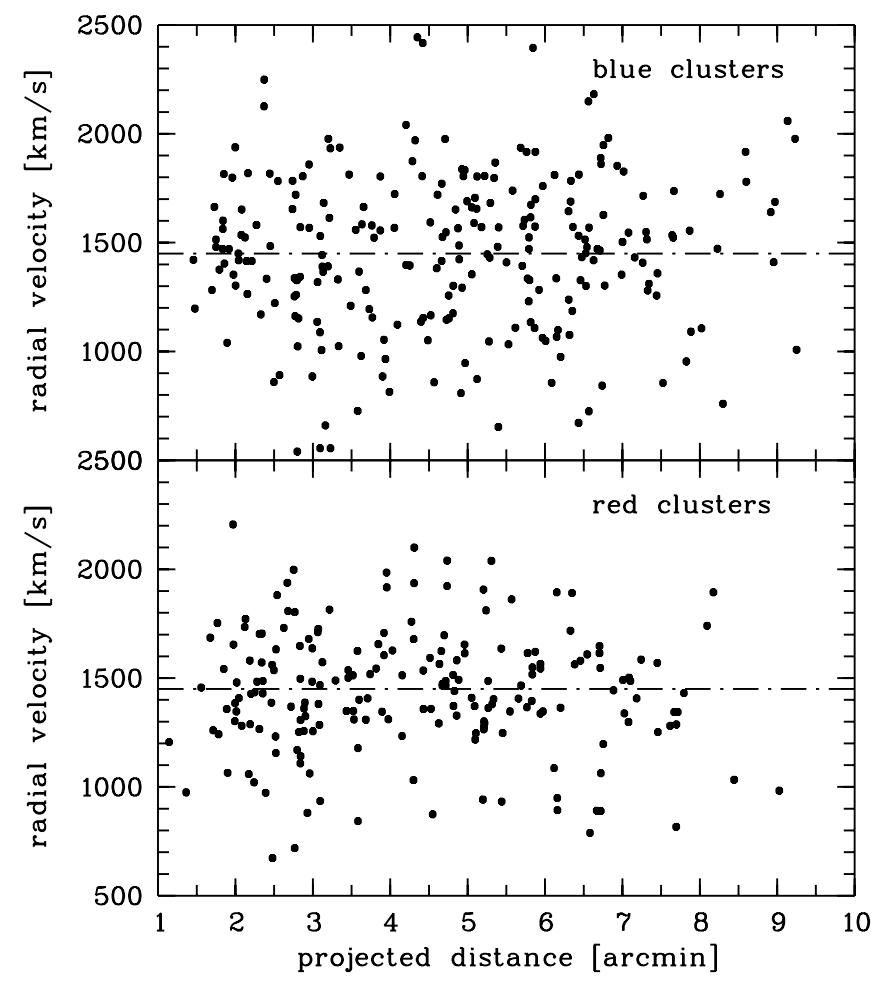

FIG. 9.-Radial velocities vs. galactocentric distance for blue and red clusters. The lower velocity dispersion of the red clusters is visible.
We select velocities between 800 and $2100 \mathrm{~km} \mathrm{~s}^{-1}$ to omit the extreme ones. Figure 10 plots the radial velocities of three samples against the azimuth angle, which goes from north to east. The top panel is the full sample, followed by the blue and blue clusters. Further selections are the outer blue clusters (more distant than $6^{\prime}$ ), the "double peak" from Figure 4 for all clusters (between 1280 and $1600 \mathrm{~km} \mathrm{~s}^{-1}$ ), and the same selection for the red clusters only, motivated by the right panel in Figure 8, where the double peak is more prominently visible than for the blue clusters. These samples are shown in Figure 11 together with the outer blue clusters. Least-squares fits for these samples return the values of $A$ and $\Theta_{0}$ listed in Table 1. As the uncertainties (the $1 \sigma$ limits) for the parameters show, only the blue outer clusters and the "peak" selection for the entire sample show rotation signals. Because of the large uncertainties, the rotation amplitude $\left(68 \pm 60 \mathrm{~km} \mathrm{~s}^{-1}\right)$ of the outer blue clusters agrees with the amplitude found by KisslerPatig et al. (1999), but not the position angle $\left(141^{\circ} \pm 39^{\circ}\right.$ east

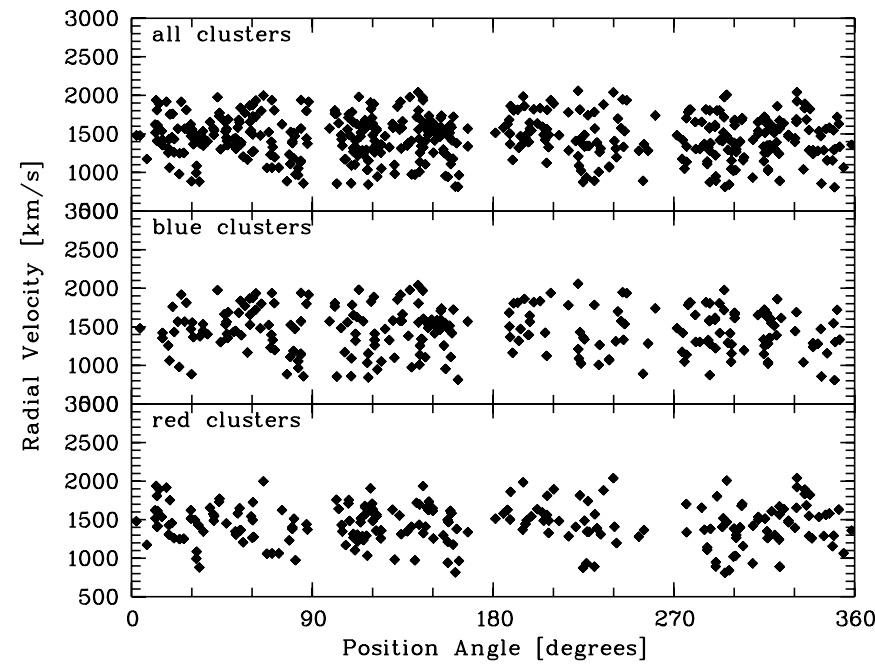

FIG. 10.-This figure is principally suitable to detect rotation. Plotted are the radial velocities vs. the azimuth angle (east past north) for the full sample (top), the red clusters (middle), and the blue clusters (bottom). There is no significant rotation present in either sample. 


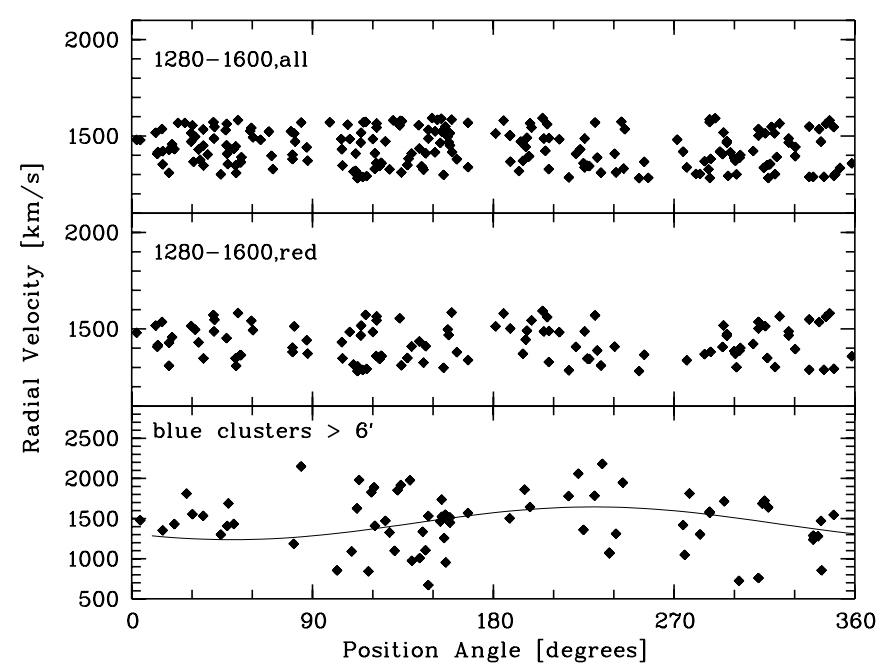

FIG. 11.-Top: Selection of velocities between 1280 and $1600 \mathrm{~km} \mathrm{~s}^{-1}$, to search for a rotation signal motivated by the double peak in Fig. 4. A rotation signal is marginally present. Middle: Same for the red clusters only, because of the more prominent double peak, but here no rotation is visible. Bottom: Blue clusters more distant than $6^{\prime}$. This sample again shows marginal rotation.

of north). These authors found a rotation amplitude of $153 \pm$ $93 \mathrm{~km} \mathrm{~s}^{-1}$ and $\Theta_{0}=210^{\circ} \pm 40^{\circ}$ (note that the authors give the position angle for the negative rotation amplitude, not $\Theta_{0}$ ). Their sample comprised 33 clusters more distant than $5^{\prime}$ from the galaxy center, without distinguishing between red and blue clusters. According to Caon, Capaccioli, \& D'Onofrio (1994), the major isophote axis of NGC 1399 is quite accurately oriented in the east-west direction. Within the uncertainties, the outer blue clusters could still rotate around the major axis.

However, the fact that the double peak formally shows a rotation signal according to equation (3) does not necessarily mean that it is indeed caused by rotation. If this were the case, then one should expect the rotation to be even more pronounced among the red clusters, where the double peak is more prominent and symmetric with respect to the systemic

TABLE 1

Amplitude and Position Angle of Possible Rotation

\begin{tabular}{|c|c|c|}
\hline Sample & $\begin{array}{c}A \\
\left(\mathrm{~km} \mathrm{~s}^{-1}\right)\end{array}$ & $\begin{array}{c}\Theta_{0} \\
(\mathrm{deg})\end{array}$ \\
\hline 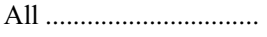 & $10 \pm 17$ & $53 \pm 110$ \\
\hline 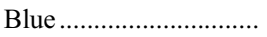 & $15 \pm 26$ & $250 \pm 102$ \\
\hline Red ................. & $7 \pm 24$ & $16 \pm 197$ \\
\hline All $\left(r>5^{\prime}\right) \ldots \ldots \ldots \ldots \ldots$ & $26 \pm 30$ & $125 \pm 56$ \\
\hline Blue $\left(r>5^{\prime}\right) \ldots \ldots \ldots \ldots . . . . . .$. & $22 \pm 40$ & $150 \pm 94$ \\
\hline $\operatorname{Red}\left(r>5^{\prime}\right) \ldots \ldots \ldots \ldots \ldots$ & $14 \pm 54$ & $253 \pm 270$ \\
\hline Blue $\left(r>6^{\prime}\right) \ldots \ldots \ldots \ldots . . . . . .$. & $68 \pm 60$ & $140 \pm 39$ \\
\hline $1280-1600$ (all)........... & $20 \pm 8$ & $198 \pm 27$ \\
\hline $1280-1600$ (red)......... & $5 \pm 12$ & $269 \pm 133$ \\
\hline
\end{tabular}

Notes.-This table lists the amplitude and the position angle of possible rotation (from north past east) for the entire radial range, for a selection with $r>5^{\prime}$, for the blue clusters with $r>6^{\prime}$, and for two further selections in the velocity interval $1280 \mathrm{~km} \mathrm{~s}^{-1}<v_{r}<1600 \mathrm{~km} \mathrm{~s}^{-1}$. The latter explores possible rotation as the cause for the double peak around the systemic velocity. Rotation around the minor axis marginally is indicated for the outer blue clusters. Although the double peak of the entire sample (Fig. 4, top left panel) yields a rotation signal, it vanishes for the red clusters in spite of being more pronounced than for the blue clusters (Fig. 8, left panel).

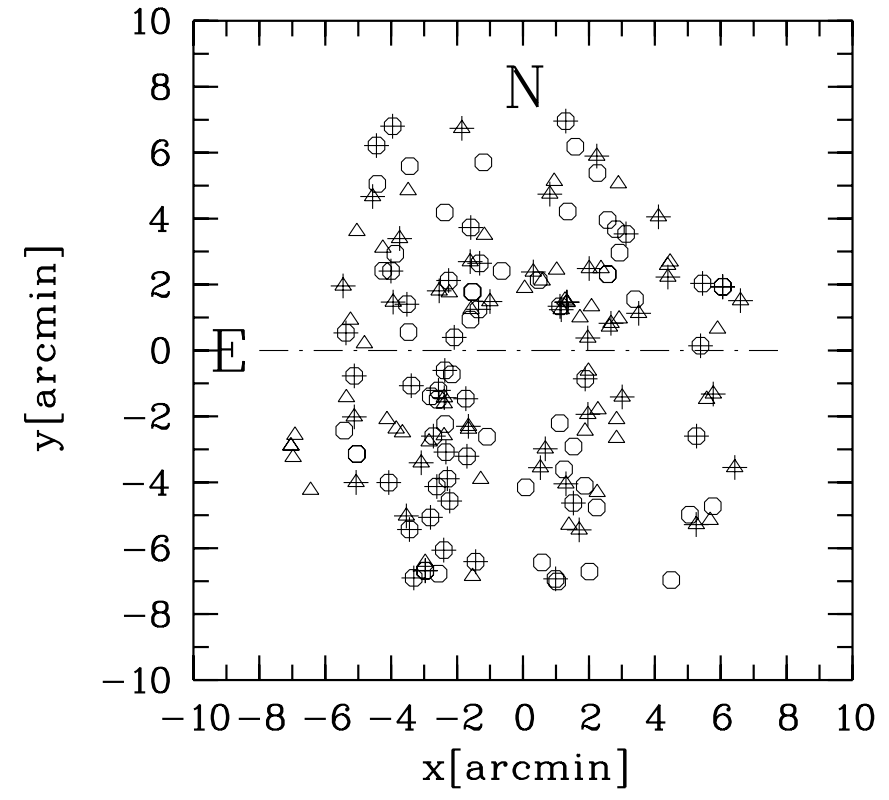

FIG. 12.-Plot of the $x y$-distribution (arcmin) for two samples referring to the two peaks near the systemic velocity. Triangles are velocities between 1280 and $1400 \mathrm{~km} \mathrm{~s}^{-1}$. Hexagons are velocities between 1480 and $1600 \mathrm{~km} \mathrm{~s}^{-1}$. Blue clusters are marked by crosses. See the text for further comments.

velocity. Figure 12 selects velocities in the intervals $1280-$ 1400 and $1480-1600 \mathrm{~km} \mathrm{~s}^{-1}$ and shows this sample in a $x-y$ plot (arcmin). The horizontal line indicates the major axis. Open hexagons and triangles show objects belonging to the lower and higher velocity intervals, respectively. Blue clusters are marked by crosses. This entire sample gives a rotation signal of $20 \pm 8 \mathrm{~km} \mathrm{~s}^{-1}$ around the major axis. However, a large part of the signal comes from the concentration of blue clusters with low velocities in the southeast quadrant, with only a few high-velocity counterparts in the northwest quadrant. The dominance of blue clusters on the east side also causes an east-west asymmetry of the double peak. On the other hand, the red clusters do not show any rotation signal, but a pronounced double peak. Generally, rotation does not appear to be significant.

The rotation pattern, or its absence, seems to constitute a difference from M87, in which apparently the entire cluster system rotates (Kissler-Patig \& Gebhardt 1998). The work of Côté et al. (2001) reveals an even more complicated pattern, in which the red clusters rotate around the photometric minor axis, while only the outer blue clusters rotate around the minor axis, and the inner blue clusters around the major axis. We refer the reader to Côté et al. for the interpretation of this result.

That only the blue clusters show signs of rotation was also observed in the NGC 4472 cluster system by Zepf et al. (2000), although with a low statistical significance. The statistics have been improved by Côté et al. (2003), who essentially confirmed Zepf et al.'s result. The metal-poor clusters rotate around the minor axis, while the metal-rich clusters do not show a significant rotation signal.

\subsection{Measurement of the Velocity Dispersions}

The most important observable that we want to extract from our data is the projected velocity dispersion and its dependence on radius. Given the above considerations regarding the extreme velocities, it is clear that neither measurement nor interpretation are completely straightforward. 


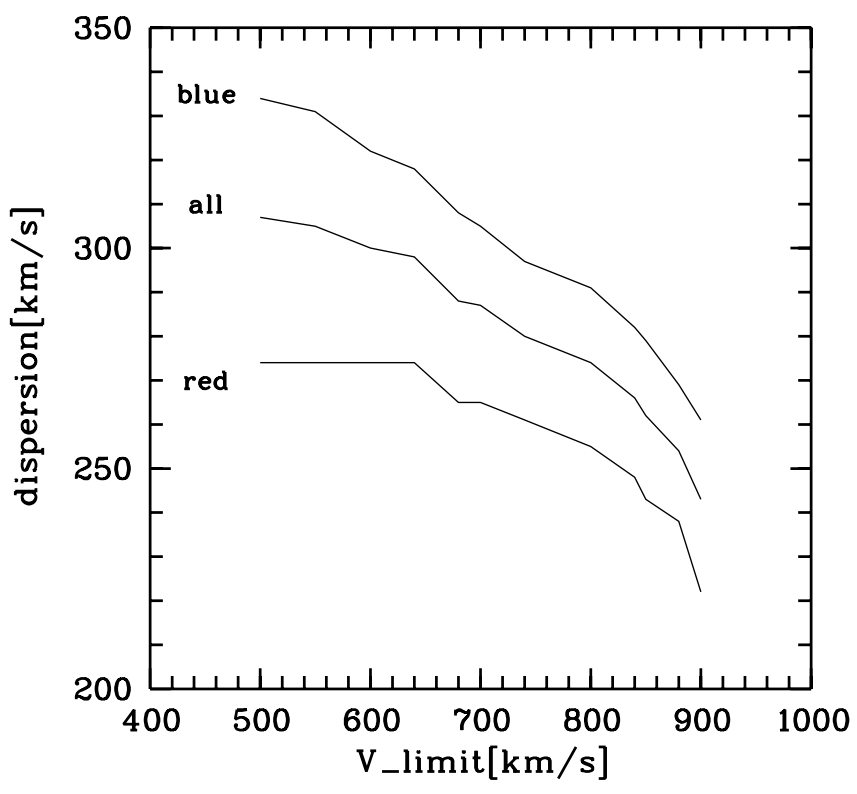

FIG. 13.-Effect of the velocity limits on the velocity dispersion for the red, the entire, and the blue sample. The $x$-axis defines the low-velocity cutoff (the high-velocity cutoff is always symmetric with respect to the systemic velocity).

We employ the maximum likelihood dispersion estimator shown by Pryor \& Meylan (1993). We fix the systemic velocity to $1441 \mathrm{~km} \mathrm{~s}^{-1}$. Then the velocity dispersion $\sigma$ is calculated (by iteration) according to

$$
\sum \frac{\left(v_{i}-v_{\mathrm{sys}}\right)^{2}}{\left(\sigma^{2}+\delta_{i}^{2}\right)^{2}}=\sum \frac{1}{\sigma^{2}+\delta_{i}^{2}}
$$

where the sum is taken over all velocities and where the $\delta_{i}$ denote the uncertainties of the individual velocities. The uncertainty of the resulting velocity dispersion is calculated according to the somewhat lengthy expression given by Pryor \& Meylan. We cannot be sure about possible contamination by objects not belonging to NGC 1399. For example, a couple of objects may be associated with nearby dwarf galaxies and with NGC 1396. Moreover, foreground objects might be present. However, removing likely nonmembers of the NGC 1399 system would require a discussion of individual objects, which we cannot give here. The global effect on the velocity dispersion will in any case be very small. This, however, is not true for the extreme velocities around 600 or $2400 \mathrm{~km} \mathrm{~s}^{-1}$. Figure 13 shows the dependence of the velocity dispersion on a lower velocity cutoff (always symmetric to the high-velocity cutoff with respect to the systemic velocity) for the total sample, the blue sample, and the red sample. Particularly for the blue clusters, the effect is not negligible. Since there is no obvious "correct" way to define which objects should be considered and which should be discarded, we refer to the bottom panel of Figure 6 and define the upper velocity limit to be $2080 \mathrm{~km} \mathrm{~s}^{-1}$ and the corresponding low limit to be $800 \mathrm{~km} \mathrm{~s}^{-1}$. However, we also show the effect of the full sample on the circular velocity and the mass profile.

We choose slightly overlapping radial bin widths of $3^{\prime}$, allowing reasonable statistics to be obtained even after subdividing the sample into blue and red clusters. Table 2 lists the results. The correlation velocities were used to derive the numbers in Table 2. Figure 14 visualizes Table 2 and shows the values of the velocity dispersion $\sigma$ versus radial distance for four samples: the unselected sample (top panel), the entire sample under velocity selection (second panel), and further selections of blue clusters (third panel) and red clusters (bottom panel). The radial bins overlap to force some smoothing, so the values are not independent. The only bin that marginally deviates from a radially constant velocity dispersion is the innermost bin of the blue clusters.

We therefore consider the projected velocity dispersion to be constant within the uncertainties. Within $3^{\prime}$ radius, there is some indication that the dispersion decreases toward the center. Using the same velocity selection, the dispersion for radial bins of $<2^{\prime}, 2^{\prime}-2 \cdot 5$, and $2{ }^{\prime} 5-3^{\prime}$ are $219 \pm 29$, $211 \pm 24$, and $292 \pm 31 \mathrm{~km} \mathrm{~s}^{-1}$, respectively. Using the entire sample, the corresponding values are $263 \pm 34,318 \pm 34$, and $331 \pm 35 \mathrm{~km} \mathrm{~s}^{-1}$. However, the sample sizes are small, and whether this decrease is real or not needs further confirmation.

The results stand in some contrast to the results of KisslerPatig et al. (1999), who suggest a strong increase between $2^{\prime}$ and $10^{\prime}$. Their radial bin samples are very small and have large uncertainties, which individually overlap with our results. Ignoring their innermost bin at $2^{\prime}$, their work also supports a constant dispersion, but at a value of $373 \pm 35 \mathrm{~km} \mathrm{~s}^{-1}$, which we would not reach even without any selection. All differences are most likely due to their small numbers, large measurement uncertainties, and inhomogeneous data. In Paper III we show that the various sources of the velocities used by Kissler-Patig et al. have considerable systematic velocity shifts. Therefore, we do not discuss these differences further.

Another point in Figure 14 is striking: the red clusters exhibit a systematically lower dispersion than the blue clusters. We show in more detail in $\S 4$ that this can be explained by the difference in their spatial density profiles.

TABLE 2

Results of the (Projected) Velocity Dispersion Measurements

\begin{tabular}{|c|c|c|c|c|c|c|c|c|}
\hline \multirow[b]{2}{*}{$\begin{array}{c}\Delta r \\
(\operatorname{arcmin})\end{array}$} & \multicolumn{2}{|c|}{ No Selection } & \multicolumn{2}{|c|}{ All Selected Clusters } & \multicolumn{2}{|c|}{ Blue Clusters } & \multicolumn{2}{|c|}{ Red Clusters } \\
\hline & $\begin{array}{c}\sigma \\
\left(\mathrm{km} \mathrm{s}^{-1}\right)\end{array}$ & $N$ & $\begin{array}{c}\sigma \\
\left(\mathrm{km} \mathrm{s}^{-1}\right)\end{array}$ & $N$ & $\begin{array}{c}\sigma \\
\left(\mathrm{km} \mathrm{s}^{-1}\right)\end{array}$ & $N$ & $\begin{array}{c}\sigma \\
\left(\mathrm{km} \mathrm{s}^{-1}\right)\end{array}$ & $N$ \\
\hline All $r \ldots \ldots \ldots \ldots$ & $325 \pm 11$ & 468 & $274 \pm 9$ & 444 & $291 \pm 14$ & 213 & $255 \pm 13$ & 213 \\
\hline$r<3.5 \ldots \ldots \ldots \ldots \ldots \ldots \ldots$ & $322 \pm 18$ & 158 & $256 \pm 15$ & 148 & $267 \pm 23$ & 71 & $246 \pm 20$ & 77 \\
\hline $2.5<r<5.5 \ldots \ldots \ldots \ldots$ & $342 \pm 16$ & 231 & $285 \pm 14$ & 219 & $307 \pm 21$ & 109 & $261 \pm 18$ & 110 \\
\hline $4.5<r<7.5 \ldots \ldots \ldots \ldots$ & $306 \pm 14$ & 234 & $267 \pm 13$ & 206 & $281 \pm 19$ & 114 & $249 \pm 18$ & 92 \\
\hline$r>6.5$ & $316 \pm 24$ & 91 & $280 \pm 22$ & 86 & $297 \pm 33$ & 47 & $258 \pm 31$ & 39 \\
\hline
\end{tabular}

Notes.-Columns list the radial bin, and the dispersion and number of objects for the entire sample, the blue clusters, and the red clusters. The uncertainties result from the expressions given by Pryor \& Meylan (1993). See the text for further comments. 


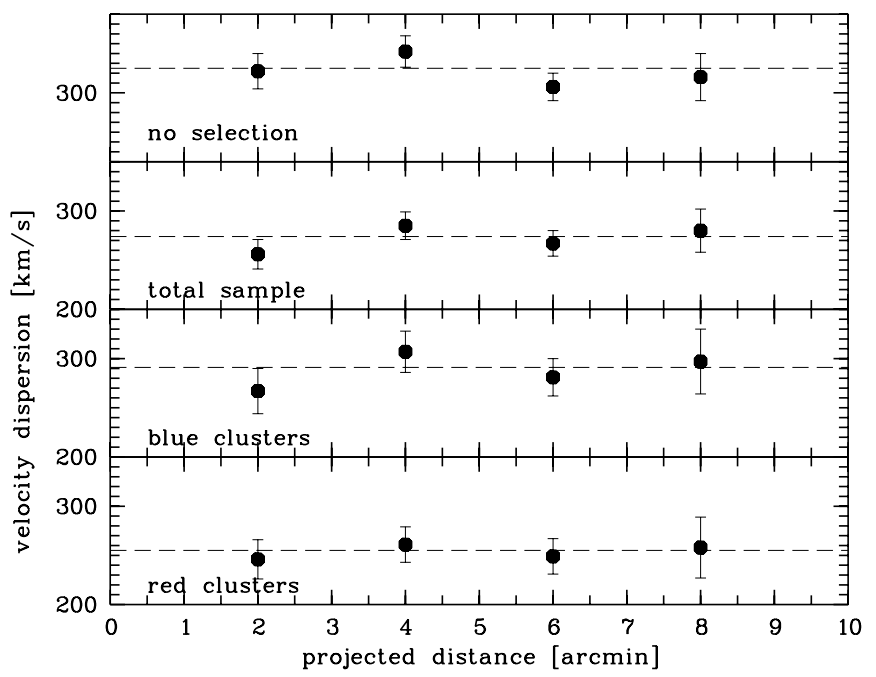

FIG. 14.-Visualization of Table 2. Plotted are the galactocentric projected distances vs. the projected velocity dispersions for the total unselected sample (top panel) and three velocity-selected samples $\left(800 \mathrm{~km} \mathrm{~s}^{-1}<v_{\text {rad }}<2080 \mathrm{~km}\right.$ $\mathrm{s}^{-1}$ ) in four radial bins: all clusters, blue clusters, and red clusters. The dashed horizontal lines indicate the velocity dispersions of the respective samples for the entire radial range. The radial behavior is consistent with a constant projected velocity dispersion. For reasons explained in the text, we consider the velocity selected sample for further analysis.

\section{DYNAMICS}

Our intention in obtaining this large velocity sample was to have a sufficient number of probes to derive the mass profile of NGC 1399 and the orbital structure simultaneously without having to make restrictive assumptions concerning spherical symmetry or isotropy. The most general models come from orbit-based axisymmetric analysis (Gebhardt et al. 2000). However, these models require a significant parameter space to explore, which we will consider in a subsequent paper (Gebhardt et al. 2004). In this paper, we first consider spherical models with different anisotropies, and discuss possible biases that this may create.

There are good reasons to believe that NGC 1399 is well approximated by a spherical and more or less isotropic model. Moreover, the fact that the projected velocity dispersion does not change with radial distance indicates that these properties also do not change significantly with radial distance. A spherical model was also used by Saglia et al. (2000) and Kronawitter et al. (2000), which allows us to make a direct comparison.

\subsection{Spherical Models}

To provide the nomenclature, we briefly introduce the spherical Jeans equation. Binney \& Tremaine (1987) showed how it can be derived from the collisionless Boltzmann equation. It reads

$$
v_{\mathrm{circ}}^{2}=\frac{G M(r)}{r}=-\sigma_{r}^{2}\left(\frac{d \ln \rho}{d \ln \mathrm{r}}+\frac{d \ln \sigma_{r}^{2}}{d \ln r}+2 \beta\right),
$$

where $v_{\text {circ }}$ is the circular velocity, $G$ is the constant of gravitation, $r$ is the galactocentric distance, $M(r)$ is the mass contained within $r, \sigma_{r}$ is the radial component of the velocity dispersion, $\rho(r)$ is the density profile of clusters, and $\beta=1-\sigma_{\Theta}^{2} / \sigma_{r}^{2}$, with $\sigma_{\Theta}$ being the tangential velocity dispersion. The azimuthal velocity dispersion $\sigma_{\Phi}$ is equal to $\sigma_{\Theta}$ in the spherical case.
Not all parameters are accessible from our observations, which only measure projected values. While we can determine $(d \ln \rho) /(d \ln r)$ from our wide-field photometry of the GCS (Paper I), we have no straightforward possibility to deproject $\sigma_{p}$, our observed projected velocity dispersion, because we only observed clusters out to $9^{\prime}$, while the wide-field photometry still finds cluster candidates at $20^{\prime}$ distance.

We assume (as all previous workers did) that NGC 1399 exhibits spherical symmetry. Strictly, this is not true. The ellipticity of NGC $1399(\epsilon=1-b / a)$ is modest, but nonzero. It ranges from about 0.1 in the inner region and may increase to 0.2 (Caon et al. 1994; Paper I).

If NGC 1399 deviates from our spherical assumption, the likely effect would be for us to underestimate the mass (an oblate elliptical, supported by an anisotropic velocity dispersion, exhibits a smaller velocity dispersion than would correspond to its mass in a spherical configuration). As Magorrian \& Ballantyne (2001) show, this situation is also expected to create a spurious radial bias. In the sample of 21 round elliptical galaxies of Gerhard et al. (2001), which they analyzed under the assumption of spherical symmetry, NGC 1399 has a central $M / L_{B}$ value of 10 , one of the highest occurring. It thus seems unlikely that this value for NGC 1399 is underestimated. However, Gerhard et al. state that no correlation is seen between their central $M / L$ values and the anisotropy. A $M / L_{B}$ value of 10 may not be surprisingly high, but so far, old metal-rich populations like the Galactic globular clusters NGC 6496 and NGC 6352 do not show the steep mass functions that are needed to produce such a value. Evaporation of low-mass stars is also not a likely explanation (Pulone et al. 2003).

We have seen that the red and blue clusters have different velocity dispersions. They must trace the same mass. If we set $\left(d \ln \sigma_{r}\right) /(d \ln r)=0$, i.e., adopting a radially constant $\sigma_{r}$, we have

$\sigma_{r, \text { red }}^{2}\left(\frac{d \ln \rho_{\text {red }}}{d \ln \mathrm{r}}+2 \beta_{\text {red }}\right)=\sigma_{r, \text { blue }}^{2}\left(\frac{d \ln \rho_{\text {blue }}}{d \ln r}+2 \beta_{\text {blue }}\right)$.

The (deprojected) density slopes are $-2.64 \pm 0.1$ and $-1.8 \pm 0.1$ for the red and the blue clusters, respectively (Paper I). For the red clusters, these numbers are valid for a larger radial range than we are considering here, while the blue clusters assume the slope of the red clusters for $r>8^{\prime}$. We see from the above equation that if $\beta$ were zero for both populations, the difference in the velocity dispersion accounts for the different radial density profiles: the equation predicts $\sigma_{r, \text { red }} / \sigma_{r, \text { blue }}=0.83$, while the observed value is 0.88 .

We cannot deproject the velocity dispersions with our still noisy data and our incomplete coverage of the cluster system. As Paper I shows, the cluster system extends to at least $100 \mathrm{kpc}$ in radius (recall that the most distant Milky Way globular clusters are beyond $100 \mathrm{kpc}$ ). However, we could get a feeling about projection effects by projecting a model cluster system with different anisotropies and requiring it to give a constant projected velocity dispersion. Doing so, we assume a cluster system with red and blue clusters, which has density profiles with power-law exponents -2.64 and -1.8 , respectively.

We use projection formula

$\sigma_{p}^{2}(R)=\frac{2}{N(R)} \cdot \int_{R}^{R_{0}} n(r) \sigma_{r}^{2}(r)\left[1-\beta(r) \frac{R^{2}}{r^{2}}\right] \frac{r d r}{\sqrt{r^{2}-R^{2}}}$,

where $\sigma_{p}$ and $\sigma_{r}$ are the projected and radial velocity dispersions, respectively, and $N(R), n(r)$ are the density profiles 


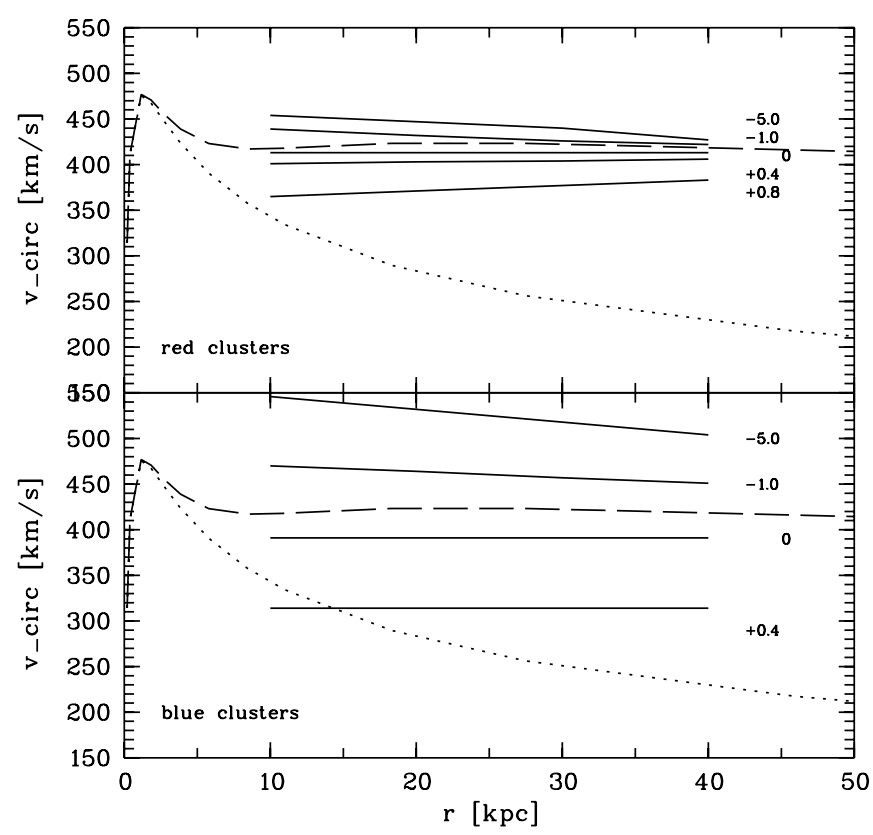

FIG. 15.-This plot is the result of projecting a model cluster system under the condition that the projected velocity dispersion is constant with radius and reproduces the observed velocity dispersions for red and blue clusters. Plotted are the resulting circular velocities vs. distance for a variety of anisotropy values of the cluster system. The dotted line comes from the luminous matter only. The long-dashed curve is the sum of the luminous mass distribution and our logarithmic potential. That the degeneracy of the red clusters is modest compared with the blue clusters is a consequence of their steeper number density profile in combination with a radially constant anisotropy.

in the same sense. Now we adopt $n(r) \sim\left(1+r / r_{c}\right)^{\alpha}$, where $r_{c}$ is a core radius, small compared with the inner limit of $10 \mathrm{kpc}$, so that a pure power-law density profile is already achieved at this radius, and $\alpha$ is an exponent which takes the above values for red and blue clusters, respectively. We also adopt an outer cutoff radius, $R_{0}$, which is set to $100 \mathrm{kpc}$, corresponding to $18^{\prime}$. This choice is arbitrary and assumes that clusters outside this radius, if they exist, do not significantly influence the projection effects. We then assume for the two values of $\alpha$ different, but radially constant values for the anisotropy in order to avoid a further extension of parameter space. The $\sigma_{r}$ is assumed to vary linearly between $10 \mathrm{kpc}$ and the cutoff radius, i.e., $\sigma_{r}=a_{1} r+a_{2}$. For the isotropic case, $\sigma_{r}$ is radially constant.

Now we ask: for each value of the anisotropy, what radial dependence is required for $\sigma_{r}$ to produce constant projected velocity dispersions of $291 \mathrm{~km} \mathrm{~s}^{-1}$ for the blue clusters and $255 \mathrm{~km} \mathrm{~s}^{-1}$ for the red clusters? After having found (by trial and error) those values of $a_{1}$ and $a_{2}$ that in projection give constant velocity dispersions within a few $\mathrm{km} \mathrm{s}^{-1}$, we then can calculate the circular velocities according to the Jeans equation.
The results of this calculation are shown in Figure 15. The top panel shows the red clusters, the bottom panel the blue clusters. The different values for $\beta$ are indicated at the righthand side ( $\beta=0.8$ has been skipped for the blue clusters). We overplot the circular velocity resulting from the luminous component alone. Table 3 lists the corresponding values of $a_{1}$ and $a_{2}$. If we assume isotropy for the red clusters, we get a constant circular velocity of $415 \mathrm{~km} \mathrm{~s}^{-1}$.

To construct a mass model for the inner region of NGC 1399 , we need the (deprojected) luminosity density profile and the assignment of a $M / L$ value, neither of which can be achieved in a straightforward manner. For the luminosity density profile we adopt the $R$ profile given in Paper I. Since the surface brightness profile itself is a composition of different observations done in different photometric bands, one has to adopt shifts depending on the color of NGC 1399 (see Paper I for details). Our adopted $R$ luminosity density profile (Kron-Cousins) is equation (2), which corresponds to a distance of $19 \mathrm{Mpc}$.

Saglia et al. (2000) quote from their dynamical modeling a $M / L_{B}$ value of 10 . To convert this into a $M / L_{R}$ value, one has to know the $B-R$ colors of NGC 1399 and the Sun (Cousins system). $B-R$ colors (in various apertures) are given by Poulain (1998; 1.56), Sandage \& Visvanathan (1978; 1.47), Mackie, Visvanathan, \& Carter (1990; 1.4), and Lauberts (1984; 1.62). We adopt a value of 1.55, omitting Mackie et al.'s measurement. For the Sun we use the theoretical $B-R$ color for the appropriate effective temperature of solar-like stars of Houdashelt, Bell, \& Sweigart (2000) and adopt $(B-R)_{\odot}=0.97$. This gives a factor of 0.59 , by which $M / L_{R}$ is smaller than $M / L_{B}$. Below we use $M / L_{R}=5.5$, taking into account the different adopted distances. Also overplotted are the total circular velocities resulting from the sum of the luminous potential and the best-fitting dark halo model (which we describe below).

The main effect of varying $\beta$ for the clusters is that in the case of a radial anisotropy $(\beta>0)$, little mass is needed to produce the required constant projected velocity dispersion, while more mass is needed when the anisotropy changes to tangential $(\beta<0)$. That this effect is larger for the blue clusters than for the red clusters is a result of their shallower density profile (the density profile and $\beta$ are additive in the Jeans equation). We note that in reality the effect of $\beta$ on the blue clusters might be slightly less than indicated in the figure. This is because the density profile of the blue clusters becomes steeper and undistinguishable from that of the red clusters (Paper I) beyond $50 \mathrm{kpc}$ of projected radial distance, while for this calculation we extrapolated the shallower profile of the inner region out to the cutoff radius.

The uncertainties of the derived circular velocities are difficult to formalize. Apart from the dominant systematic effect of the application of velocity limits, they depend on the distance, on the uncertainty of the surface density profiles, and on the

TABLE 3

Coefficients in the Adopted Linear Dependence of $\sigma_{r}$ on Galactocentric Distance

\begin{tabular}{|c|c|c|c|c|c|}
\hline \multirow[b]{2}{*}{ CoEfFicient } & \multicolumn{5}{|c|}{ Blue Clusters/Red Clusters } \\
\hline & $\beta=0.8$ & $\beta=0.4$ & $\beta=0.0$ & $\beta=-1.0$ & $\beta=-5.0$ \\
\hline$a_{1} \ldots \ldots \ldots \ldots \ldots$ & $\ldots / 0.4$ & $0.43 / 0.1$ & $0 / 0$ & $-0.35 /-0.2$ & $-0.45 /-0.22$ \\
\hline$a_{2} \ldots \ldots \ldots \ldots$ & $\ldots / 360$ & $315 / 294$ & $291 / 255$ & $245 / 205$ & $164 / 130$ \\
\hline
\end{tabular}

NotE.-Coefficients in the adopted linear dependence of $\sigma_{r}$ on galactocentric distance $\left(\sigma_{r}=a_{1} r+a_{2}\right)$, which give radially constant projected velocity dispersions for different assumed anisotropies for blue/red clusters $\left(\sigma_{r}\right.$ in $\mathrm{km} \mathrm{s}^{-1}, r$ in kpc). 
assumption that $\beta$ really is constant for the entire cluster system and varies in the adopted manner. It is easier to follow the propagation of the observational uncertainty, represented by the uncertainty of the projected velocity dispersion. To give a number, an uncertainty of $15 \mathrm{~km} \mathrm{~s}^{-1}$ translates roughly into $30 \mathrm{~km} \mathrm{~s}^{-1}$ in the circular velocity. It is then interesting to note that for the red clusters the degeneracy between mass profile and orbital structure is not more important for the derivation of the mass profile than are the observational uncertainties. In other words, a population with a steep density profile is sensitive to mass, and a population with a shallow density profile is sensitive to the orbit structure.

The above-mentioned relation between the $\beta$ values for the two populations is also visible in Figure 15. For isotropy, the blue clusters give, within the uncertainties, the same circular velocity as the red clusters. The slightly stronger tangential behavior of the blue clusters is not really significant. If the blue clusters were more tangential, then the circular velocities for $\beta=0$ would also be displaced to higher values. Thus, the different circular velocities for assumed isotropy measure the difference of the $\beta$ values. We therefore can say with some confidence that both populations are close to isotropic.

How does the analysis of the globular cluster system compare with the analysis of the inner stellar body of NGC 1399? As did Saglia et al. (2000), we use for the dark halo a logarithmic potential of the form

$$
\Phi_{\mathrm{DM}}=\frac{1}{2} v_{0}^{2} \ln \left(r^{2}+r_{0}^{2}\right)
$$

where $v_{0}$ is the asymptotic circular velocity and $r_{0}$ is the halo core radius.

Because $v_{\text {circ }}^{2}=r d \Phi / d r$, we have

$$
v_{\text {circ }}^{2}=\frac{v_{0}^{2}}{1+\left(r_{0} / r\right)^{2}} .
$$

To represent the dark halo by a logarithmic potential, we choose the circular velocities derived from the red clusters because of their lower sensitivity to $\beta$ and assume isotropy. Then we subtract the luminous component from the total mass and get the circular velocity curve for the dark halo alone at the four radii between 10 and $40 \mathrm{kpc}$ given by Figure 14. A leastsquare fit is used to obtain $v_{0}$ and $r_{0}$. We get $v_{0}=365 \pm 6 \mathrm{~km}$ $\mathrm{s}^{-1}$ and $r_{0}=11.7 \pm 0.7 \mathrm{kpc}$. The fit uncertainties are small and do not reflect the true systematic uncertainties, which are difficult to parametrize. Yet they do account for the observational uncertainty of the projected velocity dispersion. Systematic uncertainties lie in the adoption of $M / L$ and the determination of the circular velocity. Small variations of $M / L\left(\Delta M / L_{R}<1\right)$ do not change $v_{0}$ within the above uncertainties, but have a stronger effect on $r_{0}$; one gets $\Delta v_{0} / \Delta\left(M / L_{R}\right)=3$. Variations of the circular velocity change $v_{0}$ approximately by the same amount and let $r_{0}$ vary as $\Delta v_{0} / \Delta v_{\text {circ }}=-0.06$.

The "best-fitting model" of Saglia et al. (2000) has $r_{0}=$ $210^{\prime \prime}(19.3 \mathrm{kpc})$ and $v_{0}=323 \mathrm{~km} \mathrm{~s}^{-1}$ as the dark halo parameters. For other models, which also fit well the Saglia et al. data and even better to our circular velocities, no dark halo parameters are quoted.

Kronawitter et al. (2000) use the same data as Saglia et al., but different models and a different distance $(21.9 \mathrm{Mpc})$. They do not list their model parameters, but give the range of acceptable circular velocities at the last data point. These models seem to fit well, although the range still is appreciable. We note that the "best" $M / L_{B}$ of Kronawitter et al. is 10.6, i.e., larger than that of Saglia et al. in spite of the larger distance, which would lead one to expect a proportionally smaller $M / L_{B}$.

If we use this interval as reference and find those halo parameters of logarithmic potentials which span the acceptable range, we get the following:

Model 1 (high): $v_{0}=390 \mathrm{~km} \mathrm{~s}^{-1}, r_{0}=7.7 \mathrm{kpc}$.

Model 2 (best): $v_{0}=370 \mathrm{~km} \mathrm{~s}^{-1}, r_{0}=10.5 \mathrm{kpc}$.

Model 3 (low): $v_{0}=350 \mathrm{~km} \mathrm{~s}^{-1}, r_{0}=19.2 \mathrm{kpc}$.

These dark matter models, to which we add our luminous component $\left(M / L_{R}=5.5\right)$, are plotted in Figure 16 (dashed lines) together with our best model (solid line) and that of Saglia et al. (dotted line). The latter is slightly radial. The Saglia et al. model, which is almost isotropic within our radial range, has a circular velocity of $404 \mathrm{~km} \mathrm{~s}^{-1}$ at $8.9 \mathrm{kpc}$. It is encouraging to see how well the results agree in spite of the completely different analyses.

Had we renounced any velocity selection, the agreement would be worse. The unselected samples of blue and red clusters have velocity dispersions of $350 \pm 14$ and $287 \pm 15 \mathrm{~km} \mathrm{~s}^{-1}$, respectively. These values result (for isotropy) in a constant circular velocity of $465 \mathrm{~km} \mathrm{~s}^{-1}$. A fit of a logarithmic potential gives $v_{0}=412 \mathrm{~km} \mathrm{~s}^{-1}$ and $r_{0}=9 \mathrm{kpc}$. The dashed-dotted line in Figure 16 shows this model. There is no hard evidence to reject it, but the difference to the stellar models is considerable. Moreover, the increase of the circular velocity between 5 and $20 \mathrm{kpc}$ is more pronounced than in the other models. In order to have a constant projected velocity dispersion, the anisotropies of the blue and red clusters must therefore conspire. But again, the present data does not allow a firm exclusion.

\section{DISCUSSION}

\subsection{Comparison with Earlier Work on NGC 1399}

As mentioned above, there have been already some attempts to investigate the kinematics of the NGC 1399 GCS. The most

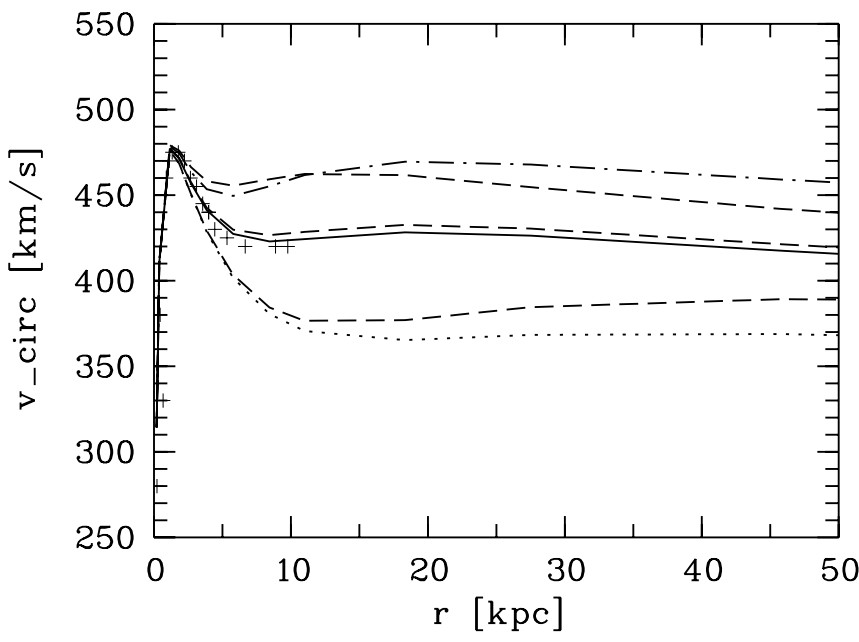

FIG. 16.-Comparison of our circular velocities (assuming isotropy for the red cluster population) with the radial extrapolation of analyses performed for the stellar body of NGC 1399. The dashed lines embrace the acceptable models of Kronawitter et al. (2000). The dotted line shows the best model of Saglia et al. (2000). Note that other models of Saglia et al., for which the halo parameters are not given, fit better. The solid line represents the circular velocities of the red cluster population assuming isotropy. The crosses mark the best solution of Kronawitter et al., read off from their circular velocity diagram. The dashed-dotted line indicates the circular velocity which would result from the full cluster sample without any velocity selection. 
recent work is that of Kissler-Patig et al. (1999), who compiled the radial velocities of 74 GCs. A comparison of their velocities in common with ours can be found in Paper III. The main finding of Kissler-Patig et al. is that the dispersion increases from about $260 \mathrm{~km} \mathrm{~s}^{-1}$ at $2^{\prime}$ to almost $400 \mathrm{~km} \mathrm{~s}^{-1}$ at $8^{\prime}$ of radial distance. The similarity of the outer velocity dispersion with that of Fornax galaxies leads them to conclude that the outer clusters might be moving in the overall Fornax potential rather than in the potential of NGC 1399. However, the uncertainties in the velocity dispersion, caused by the low number of objects and relatively large velocity uncertainties, are so large that even a constant velocity dispersion of the order of $300 \mathrm{~km} \mathrm{~s}^{-1}$ is covered by almost all of their error bars. Kissler-Patig et al. note a marked increase in the dispersion between the stellar component and the clusters. We rather say that this depends on what cluster population is considered and also what source for the stellar velocities is used. Kissler-Patig et al. use older data by Franx, Illingworth, \& Heckman (1989), Bicknell et al. (1989), and Winsall \& Freeman (1993). The more recent study by Saglia et al. (2000) finds about $250 \mathrm{~km} \mathrm{~s}^{-1}$ as the average of their outermost point, which is in excellent agreement with the red clusters, as one would expect, because this population best represents the stellar component in terms of both color and radial profile, whose power-law exponent is -1.8 (see Paper I).

The statement that the clusters are moving in the general Fornax potential can have a dubious meaning. The equality of the velocity dispersions of GCs and galaxies, however, does not say much about the dynamical relationship of these components. For example, Drinkwater et al. (2001) give the quite different values of $308 \pm 30$ and $429 \pm 41 \mathrm{~km} \mathrm{~s}^{-1}$ as the velocity dispersions for giant and dwarf Fornax galaxies, respectively (they interpret this as the difference between virialization of the giants and infall of the dwarfs).

Of course, we assume that the majority of the clusters we observed are bound to NGC 1399. However, we do not know how far the dark halo extends, and we cannot exclude the possibility that NGC 1399 is located at the bottom of a "cD dark halo" that is the central substructure in a cluster-wide dark halo (Ghinga et al. 2000). In this sense, the halo of NGC 1399 would be part of the cluster potential, but finding evidence for this is beyond present observational capacities.

Turning to planetary nebulae, Napolitano, Arnaboldi, \& Capaccioli (2002) reanalyzed older velocity data by Arnaboldi et al. (1994). In their sample of $37 \mathrm{PNe}$, they see evidence for a disturbed velocity structure and assign this to a possible past encounter with NGC 1404. We cannot comment on this apart from saying that we do not see it, but it would be very interesting to have a larger sample of $\mathrm{PNe}$ to prove or disprove it.

\subsection{The Mass Profile}

\subsubsection{General Remarks}

NGC 1399 has sometimes been labeled the central cD galaxy in the Fornax Cluster, expressing the fact that its surface luminosity falls off slower than a de Vaucouleurs law. The derived total mass profile resembles that of an isothermal sphere: its total density falls off as $r^{-2}$. Its luminous surface density in the $R$ band falls off as $r^{-1.85}$ (Paper I), which is consistent with a deprojected mass profile that can be described by a uniform power law with an exponent of $r^{-3.0}$, if the color gradient is taken into account. No feature that would indicate the onset of a cD halo is discernible. As Jaffe (1987) and White (1987) already have argued, a $r^{-4}$ profile in the outer regions of elliptical galaxies emerges if stellar orbits are scattered near the escape energy in the presence of a nearly Keplerian potential. In projection, this behavior resembles a de Vaucouleurs profile. In the case of NGC 1399, we have an isothermal sphere out to about 4 effective radii, and since the $r^{-3}$ profile of NGC 1399 is realized out to at least 10 effective radii, one may conjecture that the isothermal sphere extends to this galactocentric distance. Under this assumption, NGC 1399 would be distinguished from "normal elliptical galaxies" with a de Vaucouleurs profile by the fact that because of the huge dark halo, the Keplerian regime sets in at much larger distances than we are able to investigate, and most stars have energies much lower than the escape energy. We note that only a $r^{-3}$ profile brings into agreement the velocity dispersions derived from the Jeans equation and from the virial theorem (see van den Bosch 1999 for remarks on that matter) in the case of an isotropic tracer population in an isothermal sphere. It would also be very interesting to investigate nearby central galaxies like M87 and NGC 3311, but accurate wide-field photometry is not yet available for them.

\subsubsection{The Dark Matter Profile}

The question arises whether the dark halo of NGC 1399 could be identified with a dark halo predicted from cold dark matter (CDM) simulations. Such simulations make specific predictions regarding the structural properties, especially the density profile, of dark halos. However, in the context of galaxy formation one expects a CDM halo not to maintain its structure, but to be modified according to the dynamical details of the formation history. Therefore, our intention is not to prove or disprove CDM predictions, but to morphologically describe the dark halo in terms of parameters used in CDM simulations.

We adopt the density profile as

$$
\rho(r) \sim \frac{\rho_{s}}{\left(r / r_{s}\right)^{\zeta}\left(1+r / r_{s}\right)^{3-\zeta}},
$$

where $\rho$ is a characteristic density and $r_{s}$ a characteristic radius. The case $\zeta=1$ describes halos of the NFW type (Navarro, Frenk, \& White 1996, 1997). At small radii, its density profile is proportional to $r^{-1}$, at large radii to $r^{-3}$. Other researchers, such as Ghigna et al. (2000) and Klypin et al. (2001), performing $N$-body simulations of higher resolution, agree well regarding the outer regions, but predict steeper profiles $\left[\rho(r) \sim r^{-1.5}\right]$ near the center. For the cases where $\zeta=0$ and $\zeta=3 / 2$, the mass $M(r)$ is also analytically integrable, and we also consider them ( $\zeta=2$ does not result in a viable description).

In general, $M(r)$ becomes

$$
M(r)=4 \pi \rho_{s} r_{s}^{3} g(x),
$$

where $x=r / r_{s}$ and $g(x)$ is for the different values of $\zeta$ :

$$
g(x)= \begin{cases}\ln (1+x)+\frac{2}{1+x}-\frac{1}{2(1+x)^{2}}-\frac{3}{2} & \zeta=0, \\ \ln (1+x)-\frac{x}{1+x} & \zeta=1, \\ \frac{2}{3} \ln \left(1+x^{3 / 2}\right) & \zeta=\frac{3}{2} .\end{cases}
$$

In terms of circular velocities and convenient units,

$$
v_{\text {circ }}\left(\mathrm{km} \mathrm{s}^{-1}\right)=2.1 \times 10^{-3} \sqrt{\frac{M(r)}{r} \frac{\mathrm{kpc}}{M_{\odot}}}
$$


Adopting isotropy for the red globular cluster population (the blue cluster population then becomes slightly tangential) results in a circular velocity of $415 \mathrm{~km} \mathrm{~s}^{-1}$ between 10 and $40 \mathrm{kpc}$ of galactocentric radius. From this we subtract the luminous component and make least-square fits to the various profiles as we did for the logarithmic potential.

Within our radial range, the cases $\zeta=0$ and $\zeta=1$ fit equally well to the circular velocity curve of the dark matter. The case $\zeta=3 / 2$ fits badly, and we do not consider it further. For $\zeta=0, r_{s}$ is a core radius and $\rho_{s}$ the central density. We find $r_{s}=11.6 \pm 0.7 \mathrm{kpc}$ and $\rho_{s}=0.14 \pm 0.001 M_{\odot} \mathrm{pc}^{-3}$. For $\zeta=1$ (NFW profile), the fit results in $r_{s}=33.5 \pm 1 \mathrm{kpc}$ and $\rho_{s}=0.01 \pm 0.0006 M_{\odot} \mathrm{pc}^{-3}$. The formal fit errors only say how well the points fit to the adopted density profile, but the important uncertainties come from the uncertainties of both the circular velocities and the adopted $M / L$ of the stellar population, which we here assume to be constant, but which may well decline with radius indicated by the color gradient in NGC 1399 (Paper I).

Let us assume that we are dealing with an NFW halo. Navarro et al. $(1996,1997)$ noted that the density and the scale parameter are strongly inversely correlated. This is because small halos were formed earlier, when the universe was denser than at later times. This led to the concept of the universality of CDM halos, the halos being described by only one parameter, for instance its mass. However, other simulations (Bullock et al. 2001; Jing 2000; Jing \& Suto 2000) questioned the universality or at least showed a much larger diversity.

Following Bullock et al. (2001), more customary parameters than $\rho_{s}$ and $r_{s}$ are the virial mass $M_{\mathrm{vir}}$, which is enclosed by a virial radius $R_{\mathrm{vir}}$, inside which the mean density is by a certain factor higher than the mean universal density, depending on the cosmological model. The related velocity is $V_{\text {vir }}^{2}=G M_{\text {vir }} / R_{\text {vir }}$. For the cosmological parameters $h=0.7$, $\Omega_{m}=0.3$, and an overdensity factor of 337 (see Bullock et al. for details), handy relations are $M_{\mathrm{vir}}=0.64 \times 10^{5} R_{\mathrm{vir}}^{3}$ and $V_{\text {vir }}=0.53 \times R_{\text {vir }}$, with $M_{\text {vir }}$ in solar masses, $R_{\text {vir }}$ in kpc, and $V_{\text {vir }}$ in $\mathrm{km} \mathrm{s}^{-1}$.

In addition to $M_{\mathrm{vir}}$, the concentration $c_{\mathrm{vir}}=R_{\mathrm{vir}} / r_{s}$ is the second parameter. Then one has, in terms of circular velocities of the dark halo,

$$
V_{\text {circ }}(r)^{2}=V_{\mathrm{vir}}^{2} \frac{c_{\mathrm{vir}}}{x} \frac{g(x)}{g\left(c_{\mathrm{vir}}\right)},
$$

where $x=r / r_{s}$, and $g(x)$ is the above relation for the NFW profile (eq. [12]).

We find that a dark halo with $M_{\text {vir }}=9.7 \times 10^{12} M_{\odot}, R_{\text {vir }}=$ $533 \mathrm{kpc}$, and $c=15$ is the best representation of our circular velocity curve. Rather than giving the formal fit uncertainties (which are of the order $2 \%$ in $R_{\text {vir }}$ and $5 \%$ in $c$ ), we vary $M / L$ and the constant circular velocity of the total mass to provide estimations of how these parameters influence the halo properties. For small changes of $M / L_{R}$ up to 1 , we find $\Delta M_{\mathrm{vir}} / \Delta\left(M / L_{R}\right)=2.8 \times 10^{12}$ and $\Delta c / \Delta\left(M / L_{R}\right)=-4.5$. Varying $V_{\text {circ }}$ between 390 and $450 \mathrm{~km} \mathrm{~s}^{-1}$ has a small nonlinear effect on $M_{\mathrm{vir}}\left(9.1 \times 10^{12}<M_{\mathrm{vir}}<1.1 \times 10^{13}\right)$, and $\Delta c / \Delta V_{\text {circ }}=0.15$. For example, if $V_{\text {circ }}$ were 440 instead of $415 \mathrm{~km} \mathrm{~s}^{-1}$, the concentration parameter would change to 18 instead of 15 . These relations mean that an uncertainty of, say, $30 \mathrm{~km} \mathrm{~s}^{-1}$ in the circular velocity affects both $M_{\mathrm{vir}}$ and $c$ only modestly. The same holds true for $M / L_{R}$.

Bullock et al. (2001) show in their Figure 4 the relation between the concentration parameter and $M_{\mathrm{vir}}$ for their

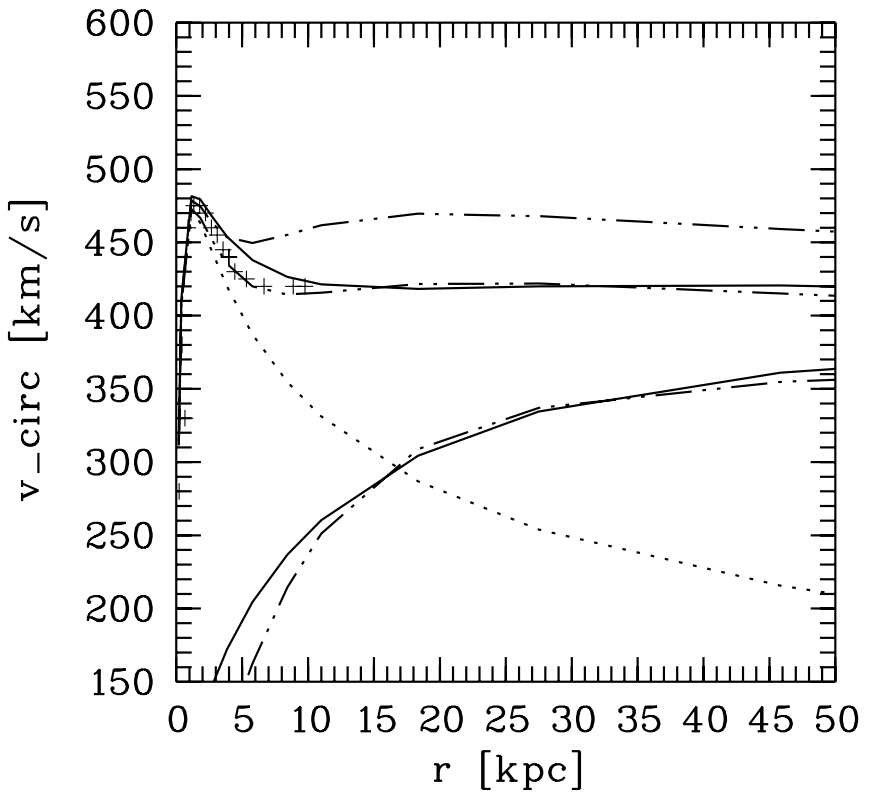

FIG. 17.- Illustration that a CDM halo can also fit the circular velocities derived from GCs. The lower dashed-dotted line corresponds to a CDM halo of the type described by Navarro et al. (1997), while the upper dashed line adds to that the luminous component (dotted line). The CDM halo has a virial mass of $9.7 \times 10^{12} M_{\odot}$ and a concentration parameter $c$ of 15 . The solid line corresponds to the sum of luminous matter (dotted line) and this halo. For comparison, a logarithmic potential with $v_{0}=365 \mathrm{~km} \mathrm{~s}^{-1}$ and $r_{0}=11.7 \mathrm{kpc}$ is plotted as well (dash-dotted line). They are indistinguishable except for the very inner region. The crosses mark the best fit of Kronawitter et al. (2000).

simulations. This plot reveals (for the Hubble parameter $h=$ 0.7 ) that a dark halo with a mass of $M_{\text {vir }}=9.7 \times 10^{12} M_{\odot}$ and the concentration $c=15$ falls more or less on the mean relation between $c$ and $M_{\text {vir }}$. It is still consistent with being a CDM halo. It is, however, obvious that galaxies like NGC 1399 with a high luminous matter density in the inner region are not very suitable for proving or disproving whether an observed dark halo resembles the type of halos found in CDM simulations.

Figure 17 shows the various circular velocity curves for these parameters. The dotted line is the luminous component, and the solid lines represent the circular velocities for the NFW halo alone and its sum with the stellar profile. The dash-dotted lines describe the same for the logarithmic potential. They are practically identical except for the inner cuspy behavior of the NFW profile. For comparison, the circular velocity curve of Kronawitter et al. (2000; their "best fit," read from their Fig. 18) is indicated by crosses. However, given the various sources of uncertainties, starting from the absolute photometric calibration to the transformation to $M / L_{R}$, one only can state that the agreement is quite good. The upper $95 \%$ confidence limit of the Kronawitter et al. profile encompasses both the NFW halo and the logarithmic potential, so one cannot distinguish between these two.

On cluster scales, dark matter halos seem to be quite consistent with NFW halos (McLaughlin 1999b; van der Marel et al. 2000). However, recent observations of brightest cluster galaxies (Kelson et al. 2002; Sand, Treu, \& Ellis 2002) resulted in much shallower dark matter profiles, which apparently challenge the universality of NFW profiles. In low surface brightness galaxies as well, de Blok \& Bosma (2002) find cores rather than the predicted cusps. 


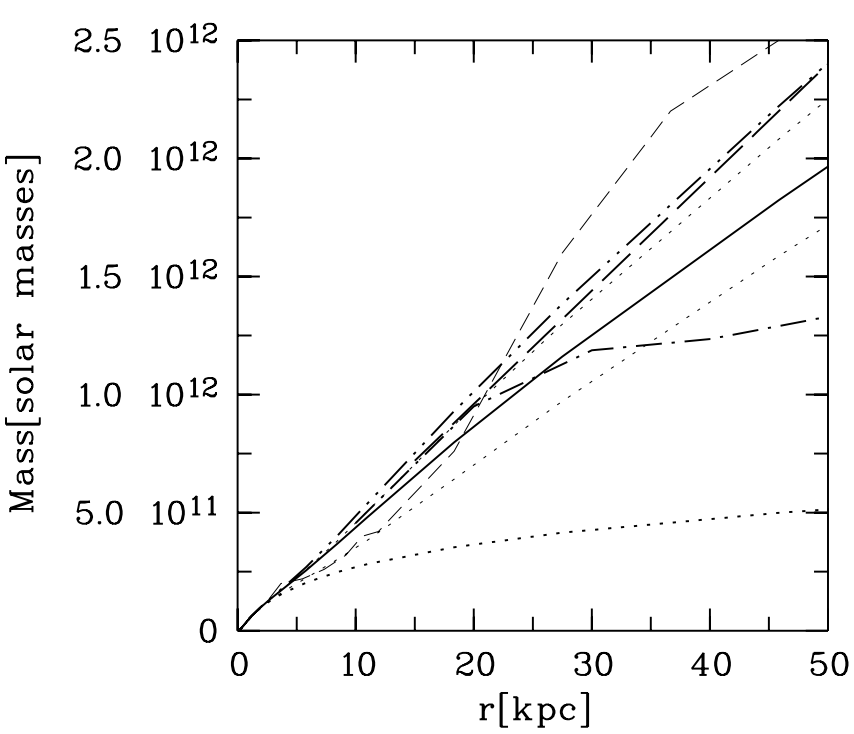

FIG. 18.-Comparison of different mass profiles of NGC 1399. The solid line is the sum of the luminous mass (lower dotted line) and a logarithmic potential with $v_{0}=365 \mathrm{~km} \mathrm{~s}^{-1}$ and $r_{0}=11.7 \mathrm{kpc}$, corresponding to a constant circular velocity of $415 \mathrm{~km} \mathrm{~s}^{-1}$. Two dotted lines indicate the mass profile if the circular velocity were 450 or $390 \mathrm{~km} \mathrm{~s}^{-1}$, respectively. The dash-dotted line shows the mass derived from ASCA X-ray data according to Ikebe et al. (1996), which is distinctly flatter. The long-dashed line shows the mass derived from ROSAT data according to Jones et al. (1997). The short-dashed line shows the mass profile according to Paolillo et al. (2002) (see text for more details), derived from ROSAT data as well. It is apparent that our mass profile does not agree with Paolillo et al. nor with Ikebe et al. The dash-dot-dotted line shows a mass profile resulting from the entire sample without any velocity selection, corresponding to a circular velocity of $465 \mathrm{~km} \mathrm{~s}^{-1}$.

\subsubsection{The Comparison with X-Ray Mass Profiles}

Figure 18 compares the various X-ray mass profiles of Jones et al. (1997; ROSAT PSPC), Ikebe et al. (1996; ASCA), and Paolillo et al. (2002; ROSAT HRI) with our logarithmic potential out to $50 \mathrm{kpc}$ of galactocentric radius. The Jones et al. profile (long-dashed line) has been obtained using their quoted formula with a constant temperature of $1.3 \mathrm{keV}$ and with a value of 1.27 for the exponent of the gas density distribution, corrected for a distance of $19 \mathrm{Mpc}$. The $A S C A$ profile (dot-dashed line) has been read from Ikebe et al.'s diagram, also assuming a linear dependence of the cumulative mass on the distance. Paolillo et al.'s profile is the short dashed line, read from their Figure 17 (power-law temperature profile). The solid line shows our mass profile for $M / L_{R}=5.5$ and $V_{\text {circ }}=415 \mathrm{~km} \mathrm{~s}^{-1}$. Two dotted lines indicate mass profiles, resulting from the assumption of circular velocities of $450 \mathrm{~km} \mathrm{~s}^{-1}$ and $390 \mathrm{~km} \mathrm{~s}^{-1}$, respectively. These values might be considered as the tolerance range. The lower dotted line is the luminous component. Since it has $M / L_{B}=10$, one reads $M / L_{B}=46$ for $50 \mathrm{kpc}$. While the ROSAT profile is in quite good agreement with our mass profile, the ASCA profile is characterized by a flattening that rises again beyond $80 \mathrm{kpc}$, suggesting to Ikebe et al. the existence of substructure in the dark matter distribution of the Fornax Cluster.

Substructure is also present in the X-ray mass profile of Paolillo et al. (2002) (Fig. 18, short-dashed line). These authors describe the X-ray halo by three components, which they label a core, a galactic, and a cluster component. Within the radial range $2^{\prime}<r<8^{\prime}$, the galactic component dominates, with a mass profile approximating $M(r) \sim r^{2}$ (their Fig. 17). This is not in agreement with our constant velocity dispersion, which implies $M(r) \sim r$. In the isotropic case, a quadratic mass dependence on radius would require $\sigma \sim r^{1 / 2}$, i.e., $\sigma$ should increase by a factor of 2 , which is ruled out. The Ikebe et al. (1996) profile predicts just the opposite, namely, a decrease of the velocity dispersion, which again we do not see. We can only suspect without having hard evidence that the nonspherical and irregular shape of the X-ray halo might be the reason for the bending of these mass profiles. It is also noteworthy that no corresponding feature is visible in the stellar luminous profile. The investigation of more distant clusters certainly would be helpful.

A comparison of Figures 18 and 15 leads to the conclusion that the global orbit distribution of the clusters should be close to isotropic, as has also been found in the cases of M87 (Côté et al. 2001) and M49 (Côté et al. 2003). We cannot really constrain the anisotropy of the red clusters, but the blue clusters trace the same mass, which narrows the permitted range of anisotropies. Even without a detailed analysis of the higher orders of the velocity distribution, a look at Figure 8 suggests that the blue clusters are more tangential than the red clusters, certainly not the opposite. A true circular velocity of, say, 450 instead of $415 \mathrm{~km} \mathrm{~s}^{-1}$ would fit even better at least to the ROSAT X-ray mass, but according to Figure 15 would cause the red clusters to gain a higher tangential bias than the blue clusters. On the other hand, lowering the circular velocity would leave the blue clusters more tangential, but enhances the difference to the X-ray mass.

\subsubsection{Extrapolation of the Mass Profile}

It is interesting to extrapolate the mass profiles of the NFW halo and the logarithmic potential toward larger galactocentric distances in order to see how they would fit into the cluster mass profile. Figure 19 shows the mass profiles out to $500 \mathrm{kpc}$.

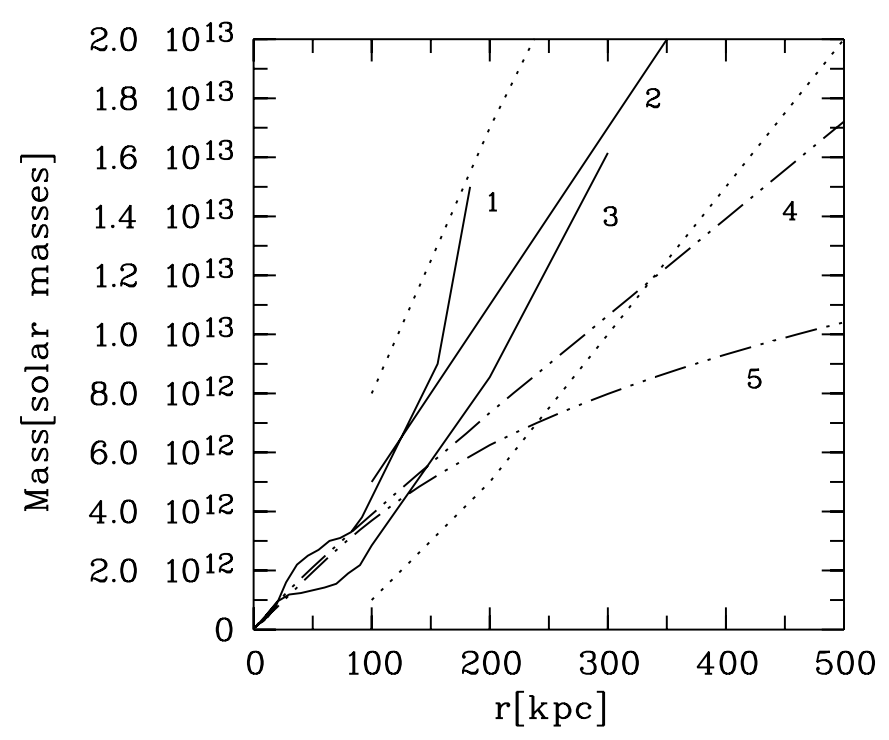

FIG. 19.-In this plot, we extrapolate our mass profiles from the inner region out to a radial distance of $500 \mathrm{kpc}$ in order to see whether such extrapolation would agree with other mass profiles, derived from galaxies or $\mathrm{X}$-rays (the shape of the X-ray profiles are approximate only, since they had to be read off from log-log plots). The labels are (1) ROSAT (Paolillo et al. 2002), (2) Drinkwater et al. (2001; the dotted lines mark their confidence limits), (3) ASCA profile (Ikebe et al. 1996), (4) logarithmic potential with $v_{0}=365 \mathrm{~km}$ $\mathrm{s}^{-1}$ and $r_{0}=11.7 \mathrm{~km} \mathrm{~s}^{-1}$, and (5) NFW profile with $r_{\mathrm{vir}}=523 \mathrm{kpc}$ and $c=15$. A comparison with the Fornax Cluster mass profile from Drinkwater et al. (2001) reveals that neither the NFW profile nor the logarithmic potential can account for the mass of the inner cluster region. The $A S C A$ profile can, but we do not duplicate its behavior in the inner region. 
The numbers 1, 2, and 3 label the ROSAT mass profile, the mass profile of the Fornax Cluster derived from galaxy velocities (Drinkwater et al. 2001), and the ASCA mass profile, respectively. Numbers 4 and 5 mark the logarithmic potential and the NFW halo. The latter two halos are hardly distinguishable out to $200 \mathrm{kpc}$. A comparison with the Fornax Cluster mass profile reveals that neither the logarithmic potential nor the NFW halo can be the dominant component of this mass, which at $500 \mathrm{kpc}$ is $(3 \pm 1) \times 10^{13} M_{\odot}$. More than 40 galaxies, roughly half of Drinkwater et al.'s sample, are projected within this radius. According to Drinkwater et al., this corresponds to $10^{11} L_{\odot}$ ( $B$ band). Even to achieve only marginal agreement, one would need for these galaxies an average $M / L_{B}$ of 100 for the NFW halo and 50 for the logarithmic potential. These are lower limits, considering that the true number of galaxies within $500 \mathrm{kpc}$ is less than the projected number. The $A S C A$ and ROSAT profiles (disregarding their mutual disagreement in shape) agree with Drinkwater et al.'s analysis. Any NFW halo that could account for the mass of this analysis is not consistent with the halo near NGC 1399.

It is possible to conceive of several reasons for this discrepancy: the dark halo of NGC 1399 may not be of the NFW type, or it may be only a substructure within the global Fornax potential, or the mass profiles may still be incorrect. The radial range $50 \mathrm{kpc}<r<100 \mathrm{kpc}$ seems to be sensitive for discriminating between the two X-ray profiles. Again, the analysis of globular cluster velocities at larger radii will be of high interest.

\subsection{Do All Objects Belong to NGC 1399?}

As already mentioned, one might suspect a few clusters to be members of dwarf galaxies close to NGC 1399. Interestingly, two of the objects with velocities higher than $2000 \mathrm{~km} \mathrm{~s}^{-1}$ seem to belong to FCCB 1241 (Ferguson 1989), which Ferguson lists as a possible member of the Fornax Cluster. General considerations regarding the extreme velocities also might lead one to suspect that not all objects are members of the NGC 1399 system.

Radial velocities below $800 \mathrm{~km} \mathrm{~s}^{-1}$ or higher than $2080 \mathrm{~km}$ $\mathrm{s}^{-1}$, which we observe out to $40 \mathrm{kpc}$ galactocentric radii, mean velocities higher than $640 \mathrm{~km} \mathrm{~s}^{-1}$ relative to NGC 1399. These velocities must belong to objects near their perigalactocentric distances. Let us assume that they are exactly at their pericenters and that we observe their space velocities (which by itself seems very unlikely). Then with the knowledge of the potential we can ask what minimal apogalactocentric distances these objects must have. Doing so, we approximate the sum of luminous and dark matter by a logarithmic potential and find that the parameters $v_{0}=400 \mathrm{~km}$ $\mathrm{s}^{-1}$ and $r_{0}=0.7 \mathrm{kpc}$ are a reasonable representation. Moreover, we assume that this potential can be extrapolated to large radii out to, say, $400 \mathrm{kpc}$.

Considering only bound orbits, conservation of energy and angular momentum gives the apocentric and pericentric velocities and radii $v_{a}, v_{p}, r_{a}$, and $r_{p}$. If $\Phi_{a}$ and $\Phi_{p}$ are the respective values of the potential, angular momentum conservation reads $r_{a} v_{a}=r_{p} v_{p}$, and energy conservation $v_{p}^{2}-v_{a}^{2}=2\left(\Phi_{a}-\Phi_{p}\right)$. This gives

$$
v_{p}^{2}=\frac{v_{p}^{2}-v_{a}^{2}}{1-\left(r_{p} / r_{a}\right)^{2}}
$$

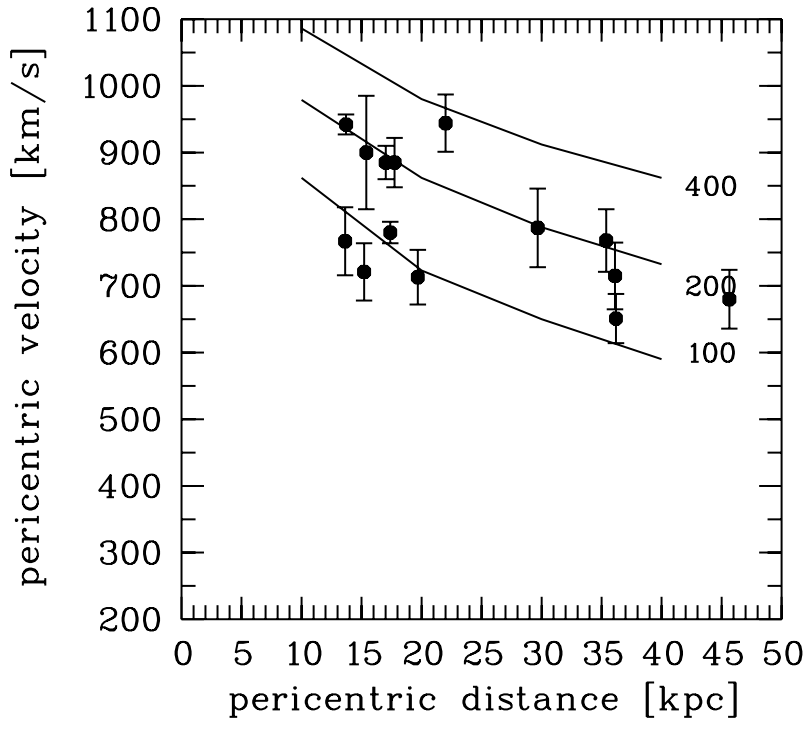

FIG. 20.-Plot of the pericenter velocities vs. the pericenter distances for bound objects with various apocentric distances (labeled in $\mathrm{kpc}$ ), which are moving in a logarithmic potential, characterized by $v_{0}=400 \mathrm{~km} \mathrm{~s}^{-1}$ and $r_{0}=0.5 \mathrm{kpc}$. This potential is a good representation of the total mass within $40 \mathrm{kpc}$ galactocentric distance, and it is assumed that it can be extrapolated out to $400 \mathrm{kpc}$. Plotted are objects of NGC 1399 with radial velocities smaller than $800 \mathrm{~km} \mathrm{~s}^{-1}$, interpreting their velocities relative to NGC 1399 as their space velocities at their pericentric positions. Already under these minimal assumptions, the majority of them must have their apocentric points at very large distances. The other possibilities are that they are not bound or that their radial velocities are recession velocities, i.e., they are in the foreground.

and

$$
v_{a}^{2}=\frac{v_{p}^{2}-v_{a}^{2}}{\left(r_{a} / r_{p}\right)^{2}-1} .
$$

For a logarithmic potential,

$$
v_{p}^{2}-v_{a}^{2}=v_{0}^{2} \ln \left(\frac{r_{0}^{2}+r_{a}^{2}}{r_{0}^{2}+r_{p}^{2}}\right) .
$$

For the above values of $v_{0}$ and $r_{0}$, Figure 20 plots perigalactic velocities versus pericentric distances for five indicated apocentric distances in kpc. Overplotted are all objects with radial velocities lower than $800 \mathrm{~km} \mathrm{~s}^{-1}$, interpreting their projected distances as pericentric distances and their radial velocities relative to NGC 1399 as pericentric velocities. These are the minimal pericentric velocities and the minimal apocentric distances. One concludes that the radius of the cluster system was at least $200 \mathrm{kpc}$, if indeed all clusters were bound to NGC 1399.

Some of them are very faint, and we have no independent confirmation by line velocities, so doubts may remain. There also remains the question of why there are not so many at the corresponding high radial velocities. Can they belong to NGC 1399? Relative velocities up to $200 \mathrm{~km} \mathrm{~s}^{-1}$ larger than the circular velocity can be easily produced by orbits with apocentric distances up to $50 \mathrm{kpc}$. But for the plotted objects, one needs distances of $200 \mathrm{kpc}$ and more. If by some strange coincidence we pick 7-8 clusters with apocentric distances of $200 \mathrm{kpc}$, why then only 4-5 with distances of $100 \mathrm{kpc}$ and not, say, 50 , considering the radial power-law number density profile of clusters? 
Can the objects with the highest relative, i.e., lowest, radial velocities be stars? The details of that discussion must be postponed, but we give an example. The object 86:105 (in the notation of Paper III) has a radial velocity of $570 \mathrm{~km} \mathrm{~s}^{-1}$, an $R$ magnitude of 20.8 , and a color of $C-R=1.2$. The most favorable case, i.e., the nearest possible distance, requires an extremely metal-poor main-sequence star with an absolute magnitude of $M_{R}=+6$, corresponding to a distance of about $9 \mathrm{kpc}$. The distance to the Galactic center then is roughly $12 \mathrm{kpc}$. The vector of Galactic rotation toward the Fornax Cluster has the value $-120 \mathrm{~km} \mathrm{~s}^{-1}$. We thus would have a star at $12 \mathrm{kpc}$ galactocentric distance with a radial velocity of $+420 \mathrm{~km} \mathrm{~s}^{-1}$ in the Galactic standard of rest and would face a similar problem as before. Given all this, we therefore call attention to the possibility that some of the objects with radial velocities lower than $800 \mathrm{~km} \mathrm{~s}^{-1}$ are objects in the foreground at an unknown distance and that their velocities have to be interpreted as recession rather than peculiar velocities.

The existence of a very diluted stellar population between the Local Group and the Fornax Cluster would be an extremely interesting matter for a number of obvious reasons. $H S T$ observations could perhaps resolve nearby objects into stars. The example cited above should have a distance of only a few Mpc. If all this were true, one would expect a comparable number per area of low-velocity objects at radii larger than $10^{\prime}$ away from NGC 1399, again a strong motivation to observe a distant cluster sample.

Another possibility is that some objects with extreme velocities are not bound to NGC 1399. Within a projected radius of $400 \mathrm{kpc}$, there are more than 20 neighboring galaxies, making it plausible that an object coming from so far is not necessarily bound. But then one should expect that there are many more unbound objects with low inclined orbits.

Of course, the mass profile of NGC 1399 has to be derived under the assumption that indeed all clusters belong to NGC 1399. It is also worth noting that the pure existence of high velocities relative to NGC 1399 requires the existence of dark matter.

\subsection{Comments on Scenarios for the Formation of the Cluster System}

In combination with the wide-field Washington study of Paper I, we currently have the largest data set available for a GCS of a giant elliptical galaxy. As we have seen, such a large sample produces a lot of new questions; but can we nevertheless obtain constraints for GCSs formation scenarios currently discussed in the literature? A compact summary of model predictions is given by Rhode \& Zepf (2001). They distinguish four different models: the "monolithic collapse" scenario (e.g., Larson 1975; Arimoto \& Yoshii 1987), the merger model put forward by Ashman \& Zepf (1992), and the models by Forbes, Brodie, \& Grillmair (1997) and Côté, West, \& Marzke (1998). The Forbes et al. model let both metal-rich and metal-poor clusters form during a dissipational collapse and involves the capture of additional GCs by tidal effects from neighboring galaxies or the accretion of dwarf galaxies. Côté et al.'s model suggests that only the metal-rich clusters have formed in a dissipational collapse, while the metal-poor clusters have been assembled hierarchically by dissipationless mergers. Rather than underlining the merits of the above suggestions (they are found in the respective papers), we want to point to a few problems which may arise when interpreting the properties of the NGC 1399 GCS in the context of any of these scenarios.
The global orbital distribution of the clusters is close to isotropic. This requires some kind of relaxation mechanism that works out to large radii. It is not plausible that a monolithic collapse model (which otherwise has been successfully applied to explain many properties of elliptical galaxies, e.g., Chiosi \& Carraro 2002 and references therein), can provide such a mechanism. Moreover, in this model, one would expect rotation to be more pronounced in the inner regions, while there is only a weak signal for the outer blue clusters. A further argument from the photometric work is that the local specific frequency of the clusters is increasing outward. On the other hand, there is evidence that it depends on the star formation rate (see remarks below). Therefore, if the star formation rate increases inward according to the gas density profile, one would expect to see the cluster formation efficiency increasing inward as well (Larsen \& Richtler 1999, 2000). This should not result in an outward-increasing local specific frequency, as is the case in NGC 1399 (Paper I), unless cluster destruction processes significantly shaped the cluster number density profile in our radial range. This, however, is unlikely. One would then expect an increasing tangential bias for both red and blue clusters, for which there is no evidence.

We note that the remark on the uncertain anisotropy also applies to the analysis of Côte et al. (2001) of the M87 cluster system. They find the red clusters to have $\beta=+0.4$ and the blue clusters to have $\beta=-0.4$, when they impose the mass profile taken from X-ray analyses. While the red clusters probably always stay more radial, both the absolute values and the difference are uncertain and may change strongly when the mass profile is changed only moderately. A similar approach in the case of NGC 1399 would have to face the contradicting mass profiles and therefore is prohibited, unless there is better agreement.

Can the blue clusters be accreted, as in the scenario of Côté et al. (1998)? Invented primarily to explain the phenomenon of bimodality, we note that one of its predictions, namely the high specific frequency of central galaxies, is questioned by the fact that NGC 1399 is probably more "normal" in its specific frequency than previously thought (Paper I; Ostrov et al. 1998). However, Côté et al. refer to M87, for which a wide-field study has yet to be done. Furthermore, Côté et al. do not expect to find a bimodal GC color distribution in dwarf ellipticals, while there is now the example of NGC 1427, a low-luminosity elliptical with bimodality (Forte et al. 2001). They also cite the near equality of the velocity dispersion of outer globular clusters with Fornax galaxies found by KisslerPatig et al. (1999) as evidence that the blue clusters are tidal debris. Our velocity dispersions are indeed similar to that of the Fornax giant ellipticals, but distinctly lower than those of the dwarf galaxies. Drinkwater et al. (2001) quote $308 \pm$ $30 \mathrm{~km} \mathrm{~s}^{-1}$ for giants and $429 \pm 41 \mathrm{~km} \mathrm{~s}^{-1}$ for dwarfs.

Globular clusters cannot be accreted individually, for example from a free-floating intergalactic population, because neither dynamical friction nor tidal interaction with the accreting host are sufficient for those compact and low-mass objects to create a strongly bound population. They have to be brought in, as in the scenario of Côté et al. (1998), as members of the cluster system of an infalling galaxy. The question is whether the above processes are able to produce an isotropic or slightly tangential orbit distribution of GCs at galactocentric radii between 10 and $40 \mathrm{kpc}$. Trustworthy answers must rely on $N$-body simulations. What we qualitatively can take from such simulations (e.g., van den Bosch 
1999) is that infalling satellites of sufficient mass on circular orbits spiral inward on a timescale of about a few Gyr. But such satellites (model 6 of van den Bosch et al. has a mass of $2 \times 10^{10} M_{\odot}$ ) are not tidally destroyed until they reach small perigalactic distances, so they should still be visible. Tidal disruption occurs effectively on strongly elongated orbits (Seguin \& Dupraz 1996), where circularization of the orbits can occur (Tormen, Diaferio, \& Syer 1998). However, clusters tidally released from those satellites and now found again at large galactocentric distances must have been released at a very early stage in order to maintain their highly eccentric orbits. In total, one would expect a radial bias and not isotropy or even a tangential bias. Moreover, one would not expect to see any rotation, as possibly seen for the outer blue clusters. It thus seems improbable that a large fraction of the blue clusters have been accreted through the infall of dwarf galaxies.

A kinematic study of the clusters at larger galactocentric radii may turn out to be more discriminating between the collapse, infall, or merger scenarios. If the rotation of the outer blue clusters is confirmed, an infall scenario becomes less probable, while rotation can be understood as a relic of a major merger event, as in the case of M87 (Kissler-Patig \& Gebhardt 1999; Côté et al. 2001).

\section{CONCLUSIONS AND OUTLOOK}

We obtained radial velocities for 468 globular clusters of the globular cluster system of NGC 1399, the central galaxy in the Fornax Cluster. The clusters have projected radial distances between $2^{\prime}$ and $9^{\prime}$, corresponding to 11 and $50 \mathrm{kpc}$. The main results emerging from this study are:

We do not find any signature of rotation except for the outer metal-poor clusters, where a marginal rotation amplitude might be present.

The velocity distribution of the clusters is not easy to interpret. Particularly under error selection, one finds an asymmetry with respect to the systemic velocity $\left(1441 \mathrm{~km} \mathrm{~s}^{-1}\right)$. There are more objects with extreme low velocities down to $600 \mathrm{~km} \mathrm{~s}^{-1}$ than in the high-velocity wing. A strong error selection produces velocity limits at radial velocities symmetric to the systemic velocity, suggesting that there are indeed only very few objects with velocities larger than $2080 \mathrm{~km} \mathrm{~s}^{-1}$ or lower than $800 \mathrm{~km} \mathrm{~s}^{-1}$. We suggest that a foreground population of sources may contaminate the lowvelocity sample. Another possibility is that they are not bound to NGC 1399.

When omitting the extreme velocities, the projected velocity dispersion of the sample is $274 \pm 9 \mathrm{~km} \mathrm{~s}^{-1}$. It remains radially constant within the uncertainties. For the nonselected sample, it rises to $325 \pm 11 \mathrm{~km} \mathrm{~s}^{-1}$, while still remaining radially constant. This constancy stands in contrast to earlier investigations with smaller samples, which found a strong radial increase of the velocity dispersion.
Dividing our sample into red (metal-rich) and blue (metalpoor) clusters, we find a velocity dispersion of $255 \pm 13$ for the red clusters and $291 \pm 14$ for the blue clusters, both radially constant. This difference is in agreement with the assumption of isotropic orbits and the observed surface density distributions.

We adopted a spherical dynamical model on the basis of the radial Jeans equation, and tried to constrain $\sigma_{r}$, the radial component of the velocity dispersion. By projecting a model globular cluster system, we obtained circular velocity curves, which depend on the anisotropy, which we cannot determine directly. However, for the red clusters the anisotropy does not influence the circular velocity much. Adopting isotropy, we derive a radially constant circular velocity of $415 \pm 30 \mathrm{~km} \mathrm{~s}^{-1}$.

If a $M / L_{R}$ value of 5.5 for the stellar component is adopted, the dark matter component is well described by a logarithmic potential with the parameters $v_{0}=365 \pm 6 \mathrm{~km} \mathrm{~s}^{-1}$ and $r_{0}=$ $11.7 \pm 0.7 \mathrm{kpc}$ (the small error bars tell us that the shape of a logarithmic potential is a very good representation. The dominant uncertainty still is the observed velocity dispersion). Within our radial range, this potential is indistinguishable from a CDM halo of the NFW type with a virial mass of $9.7 \times 10^{12} M_{\odot}$ and a concentration parameter $c=15$ (using the definitions of Bullock et al. 2001). Observations of other central galaxies revealed much shallower density profiles.

Our mass profile disagrees with the X-ray analysis of Paolillo et al. (2002), which results in a mass profile increasing with $\sim r^{2}$ instead of increasing linearly. One might suspect that the irregular shape of the X-ray halo is somehow related to this disagreement. It also (although less certainly) cannot duplicate the mass profile of Ikebe et al. (1996), which predicts the opposite, namely, a shallower profile.

Although our sample of radial velocities is the largest so far obtained for any elliptical galaxy, important questions remain: is there really an asymmetry in the extreme velocities, or would it disappear in a larger sample? Is there rotation? Out to what radius can the constant circular velocity be followed? The important next step should be to investigate the outer cluster population beyond $40 \mathrm{kpc}$.

Thanks are due to Petra Gärtner for help with the reductions of some masks. We are indebted to Ylva Schuberth for many helpful remarks. The comments of the referee, Dean McLaughlin, were quite essential for improving the first version of the paper. T. R. and D. G. acknowledge ESO visitorships, during which some of the work has been done. B. D. thanks the Humboldt Science Foundation, Germany, for financial support. T. R., B. D., D. G., D. M., and L. I. acknowledge support from the FONDAP center for astrophysics, CONICYT 15010003.

\section{REFERENCES}

Arimoto, N., \& Yoshii, Y. 1987, A\&A, 173, 23

Arnaboldi, M., Freeman, K., Hui, X., Capaccioli, M., \& Ford, H. 1994, Messenger, 76, 40

Ashman, K. M., \& Zepf, S. E. 1992, ApJ, 384, 50

Bender, R., Saglia, R. P., \& Gerhard, O. 1994, MNRAS, 269, 785

Bicknell, G. V., Carter, D., Killen, N. E., \& Bruce, T. E. 1989, ApJ, 336, 639

Binney, J., \& Tremaine, S. 1987, Galactic Dynamics (Princeton: Princeton Univ. Press)

Bridges, T. J., Hanes, D. A., \& Harris, W. E. 1991, AJ, 101, 469

Bullock, J. S., et al. 2001, MNRAS, 321, 559

Buote, D. A. 2000, MNRAS, 311, 176
Buote, D. A. 2002, ApJ, 574, L135

Caon, N., Capaccioli, M., \& D’Onofrio, M. 1994, A\&AS, 106, 199

Chiosi, C., \& Carraro, G. 2002, MNRAS, 335, 335

Cohen, J. G. 2000, AJ, 119, 162

Cohen, J. G., \& Ryzhov, A. 1997, ApJ, 486, 230

Côté, P., McLaughlin, D., Cohen, J. G., \& Blakeslee, J. P. 2003, ApJ, 591, 850

Côté, P., et al. 2001, ApJ, 559, 828

Côté, P., West, M. J., \& Marzke, R. 1998, ApJ, 501, 554

Da Costa, L. N., et al. 1998, AJ, 116, 1

de Blok, W. J. G., \& Bosma, A. 2002, A\&A, 385, 816

Dejonghe, H. B. 1987, MNRAS, 224, 13 
de Vaucouleurs, G., et al. 1991, Third Reference Catalogue of Bright Galaxies (New York: Springer)

de Zeeuw, T. 1994, in The Formation and Evolution of Galaxies, ed. C. Muñoz-Tuñón \& F. Sánchez (Cambridge: Cambridge Univ. Press)

Dirsch, B., et al. 2003, AJ, 125, 1908 (Paper I) 2004, AJ, in press (Paper III)

Drinkwater, M. J., Gregg, M. D., \& Colless, M. 2001, ApJ, 548, L139

Drinkwater, M. J., Gregg, M. D., Holman, B. A., \& Brown, M. J. I. 2001, MNRAS, 326, 1076

Elmegreen, B. 2000, in Towards a New Millenium in Galaxy Morphology, ed. D. L. Block et al. (Dordrecht: Kluwer)

Fabricant, D., \& Gorenstein P. 1983, ApJ, 267, 535

Ferguson, H. C. 1989, AJ, 98, 367

Ferrarese, L., et al. 2000, ApJ, 529, 745

Forbes, D. A., Beasley, M. A., \& Brodie, J. P. 2001, ApJ, 563, L143

Forbes, D. A., Brodie, J. P, \& Grillmair, C. J. 1997, AJ, 113, 1652

Forbes, D. A., et al. 1998, MNRAS, 293, 325

Foreman, W., Jones, C., \& Tucker, W. 1985, ApJ, 293, 102

Forte, J. C., Geisler, D., Ostrov, P., Piatti, A. E., \& Gieren, W. 2001, AJ, 121, 1992

Franx, M., Illingworth, G., \& Heckman, T. 1989, AJ, 98, 538

Gebhardt, K., et al. 2004, in preparation 2000, AJ, 119, 1157

Gerhard, O. 1993, MNRAS, 265, 213

Gerhard, O., Kronawitter, A., Saglia, R. P., \& Bender, R. 2001, AJ, 121, 1936

Ghigna, S., et al. 2000, ApJ, 544, 616

Graham, A. W., et al. 1998, A\&AS, 133, 325

Grillmair, C., et al. 1994, ApJ, 422, L9

Hanes, D., et al. 2001, ApJ, 559, 812

Harris, W. E. 2001, in Star Clusters, Saas-Fee Advanced Course 28, ed.

L. Labhardt \& B. Binggeli (Berlin: Springer), 223

Harris, W. E., \& Harris, G. H. 2002, AJ, 123, 3108

Harris, W. E., \& van den Bergh, S. 1981, AJ, 86, 1627

Hilker, M., Infante, L., \& Richtler, T. 1999, A\&AS, 138, 55

Houdashelt, M., Bell, R. A., \& Sweigart, A. V. 2000, AJ, 119, 1448

Ikebe, Y., et al. 1996, Nature, 379, 427

Irwin, J. A., \& Sarazin, C. L. 1996, ApJ, 471, 683

Jaffe, W. 1987, in IAU Symp. 127, Structure and Dynamics of Elliptical Galaxies, ed. T. de Zeeuw (Dordrecht: Reidel), 511

Jing, Y. P. 2000, ApJ, 535, 30

Jing, Y. P., \& Suto, Y. 2000, ApJ, 529, L69

Jones, C., Stern, C., Forman, W., Breen, J., David, L., Tucker, W., \& Franx, M. 1997, ApJ, 482, 143

Kelson, D. D., Zabludoff, A. I., Williams, K. A., Trager, S. C., Mulchaey, J. S., \& Bolte, M. 2002, ApJ, 576, 720

Kissler, M., Hilker, M., Richtler, T., Infante, L., \& Quintana, H. 1997, A\&A, 319,470

Kissler, M., Richtler, T., Held, E., Grebel, E. K., Wagner, S. J., \& Capaccioli, M. 1994, A\&A, 287, 463

Kissler-Patig, M., \& Gebhardt, K. 1998, AJ, 116, 2237

Kissler-Patig, M., Grillmair, C. J., Meylan, G., Brodie, J. P., Minniti, D., Goudfrooij, P. 1999, AJ, 117, 1206

Klypin, A., Kravtsov, A., Bullock, J. S., \& Primack, J. R. 2001, ApJ, 554, 903

Kronawitter, A., Saglia, R. P., Gerhard, O., \& Bender, R. 2000, A\&AS, 144, 53

Kundu, A., \& Whitmore, B. C. 2001, AJ, 121, 2950
Larsen, S. S., Brodie, J. P., Huchra, J. P., Forbes, D. A., \& Grillmair, C. J. 2001, AJ, 121, 2974

Larsen, S. S., \& Richtler, T. 1999, A\&A, 345, 59 2000, A\&A, 354, 836

Larson, R. B. 1975, MNRAS, 173, 671

Lauberts, A. 1984, A\&AS, 58, 249

Mackie, G., Visvanathan, N., \& Carter, D. 1990, ApJS, 73, 637

Magorrian, J., \& Ballantyne, D. 2001, MNRAS, 322, 702

Matilsky, T., Jones, C., \& Forman, W. 1985, ApJ, 291, 621

McLaughlin, D. E. 1999a, AJ, 117, 2398 1999b, ApJ, 512, L9

Méndez, R. A., Riffeser, A., Kudritzki, R.-P., Matthias, M., Freeman, K. C., Arnaboldi, M., Capaccioli, M., \& Gerhard, O. E. 2001, ApJ, 563, 135

Merritt, D., \& Tremblay, B. 1993, AJ, 106, 2229

Mieske, S., Hilker, M., \& Infante, L. 2002, A\&A, 383, 823

Mould, J. R., Oke, J. B., de Zeeuw, P. T, \& Nemec, J. 1990, AJ, 99, 1823

Mushotzky, R., Loewenstein, M., Awaki, H., Makishima, K., Matsushita, K., \& Matsumoto, H. 1994, ApJ, 436, L79

Napolitano, N.R., Arnaboldi, M., \& Capaccioli, M. 2002, A\&A, 383, 791

Navarro, J. F., Frenk, C. S., \& White, S. D. M. 1996, ApJ, 462, 563 1997, ApJ, 490, 493

Nulsen, P. E. J., \& Böhringer, H. 1995, MNRAS, 274, 1093

Ostrov, P. G., Forte, J. C., \& Geisler, D. 1998, AJ, 116, 2854

Paolillo, M., Fabbiano, G., Peres, G., \& Kim, D.-W. 2002, ApJ, 565, 883

Poulain, P. 1988, A\&AS, 72, 215

Pryor, C., \& Meylan, G. 1993, in ASP Conf. Ser. 50, Structure and Dynamics of Star Clusters, ed. S. G. Djorgovski \& G. Meylan (San Francisco: ASP), 357

Pulone, L., DeMarchi, G., Covino, S., \& Paresce, F. 2003, A\&A, 399, 121

Rhode, K. L., \& Zepf, S. E. 2001, AJ, 121, 210

Richtler, T., Drenkhahn, G., Gómez, M., \& Seggewiss, W. 1999, in From Extrasolar Planets to Cosmology: The VLT Opening Symposium, ed. J. Bergeron \& A. Renzini (Berlin: Springer), 259

Rix, H. W., de Zeeuw, P. T., Cretton, N., van der Marel, R. P., \& Carollo, C.M. 1997, ApJ, 488, 702

Saglia, R. P., Kronawitter, A., Gerhard, O., \& Bender, R. 2000, AJ, 119, 153

Sand, D. J., Treu, T., \& Ellis, R. J. 2002, ApJ, 574, L129

Sandage, A., \& Visvanathan, N. 1978, ApJ, 223, 707

Seguin, P., \& Dupraz, C. 1996, A\&A, 310, 757

Sofue, Y., \& Rubin, V. 2001, ARA\&A, 39, 137

Tonry, J. L., Dressler, A., \& Blakeslee, J. P. 2001, ApJ, 546, 681

Tormen, G., Diaferio, A., \& Syer, D. 1998, MNRAS, 299, 728

van den Bosch, F. C., Lewis, G. F., Lake, G., \& Stadel, J. 1999, ApJ, 515, 50

van der Marel, R., \& Franx, M. 1993, ApJ, 407, 525

van der Marel, R. P., Magorrian, J., Carlberg, R. G., Yee, H. K. C., \& Ellingson, E. 2000, AJ, 119, 2038

Wagner, S., Richtler, T., \& Hopp, U. 1991, A\&A, 241, 399

Wegner, G., et al. 2003, AJ, submitted

White, S. 1987, in IAU Symp. 127, Structure and Dynamics of Elliptical Galaxies, ed. T. de Zeeuw (Dordrecht: Reidel), 339

Winsall, M. L., \& Freeman, K. C. 1993, A\&A, 268, 443

Woodworth, S. C., \& Harris, W. E. 2000, AJ, 119, 2699

Zepf, S., Beasley, M. A., Bridges, T. J., Hanes, D. A., Sharples, R. M., Ashman, K. M., \& Geisler, D. 2000, AJ, 120, 2928 